\title{
THE SCHRÖDINGER DENSITY AND THE TALBOT EFFECT
}

\author{
K. I. OSKOLKOV \\ Department of Mathematics, University of South Carolina \\ Columbia, SC 29208, U.S.A. \\ E-mail: oskolkov@math.sc.edu
}

\begin{abstract}
We study the local properties of the time-dependent probability density function for the free quantum particle in a box, i.e. the squared magnitude of the solution of the Cauchy initial value problem for the Schrödinger equation with zero potential, and the periodic initial data. $\sqrt{\delta}$-families of initial functions are considered whose squared magnitudes approximate the periodic Dirac $\delta$-function. The focus is on the set of rectilinear domains where the density has a special character, in particular, remains bounded, or even has low average values ("the valleys of shadows").

An essential part of the paper is dedicated to a review of some earlier results concerning the fractal properties of Vinogradov's extensions, which incorporate the solutions of a wide class of Schrödinger type equations. Relations are discussed with the optical diffraction phenomena discovered in 1836 by W. H. F. Talbot, the British inventor of photography. In the modern Physics literature, self-similarity in the wave diffracted by periodic gratings, is known as fractional and fractal revivals, and quantum carpets (M. Berry, W. Schleich, and many others). Self-similarity has been well-known, and extensively utilized in Analytic Number Theory, since the creation of the circle method of Hardy-Littlewood-Ramanujan, and Vinogradov's method of estimation and asymptotic formulas for H. Weyl's exponential sums. According to these methods, on the major arcs, the complete rational exponential sums are the scaling factors, while the appropriate oscillatory integrals constitute the pattern of the arising arithmetical carpets.
\end{abstract}

1. Introduction. Some time ago, in a personal conversation, Z. Ciesielski advised me to study the properties of the solutions of the Cauchy initial value problem for the Schrödinger equation of a free particle, with the Jacobi elliptic $\vartheta$-function as the pe-

2000 Mathematics Subject Classification: 42A16, 35J10, 11L017, 11T24, 81.

Key words and phrases: Cauchy initial value problem, Schrödinger equation, Wigner's function, Helmholtz equation, exponential sums, circle method, Gauss' sums, density, self-similarity, Hilbert transforms, asymptotical formulas, quadratic variation.

The paper is in final form and no version of it will be published elsewhere. 


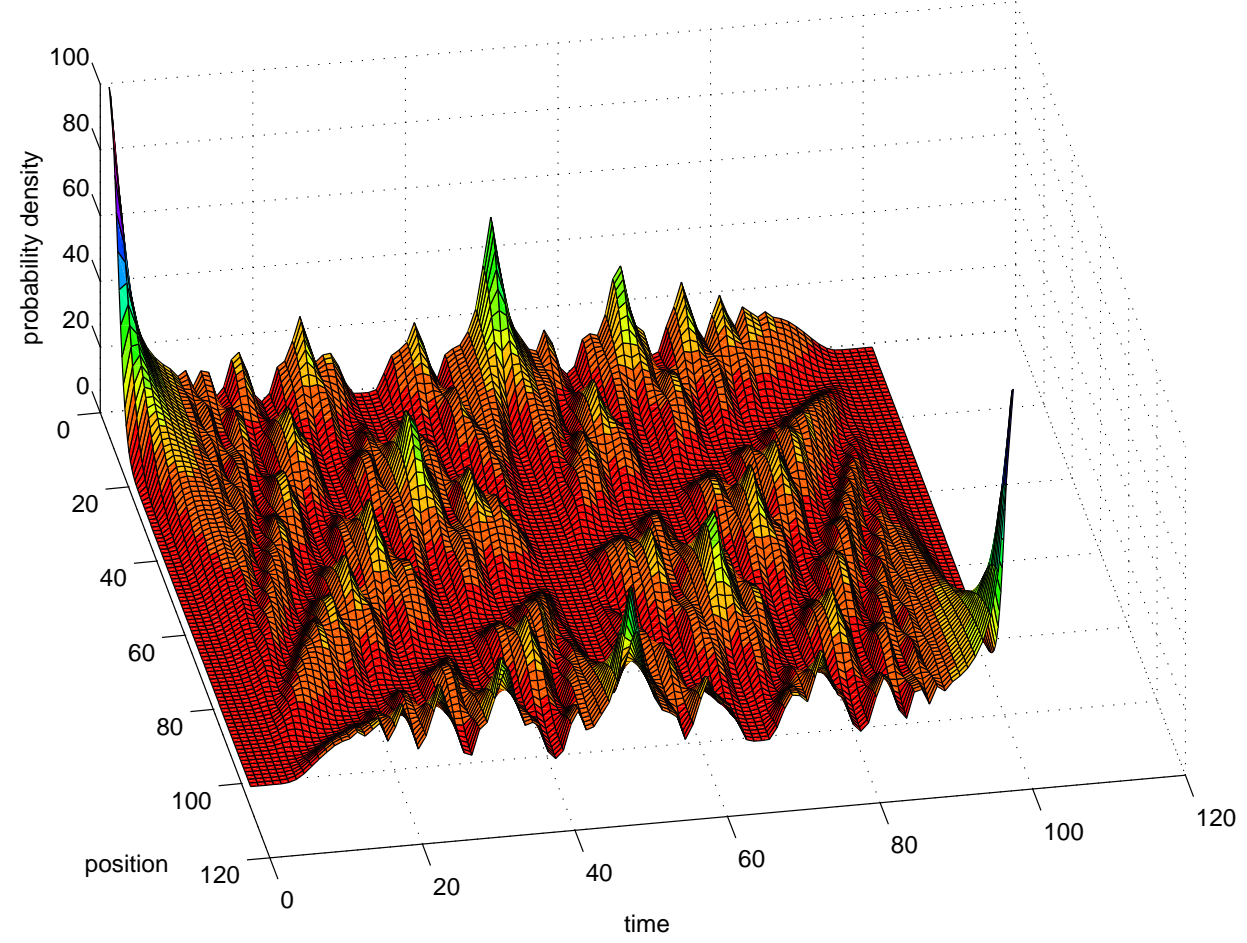

Fig. 1. The Schrödinger landscape

riodic initial data:

$$
\frac{\partial \psi}{\partial t}=\frac{1}{2 \pi i} \frac{\partial^{2} \psi}{\partial x^{2}},\left.\quad \psi(t, x)\right|_{t=0}=\vartheta_{\varepsilon}(x)=c(\varepsilon) \sum_{m \in \mathbb{Z}} e^{-\frac{\pi(x-m)^{2}}{\varepsilon}} .
$$

Here, $\varepsilon$ is a small positive number (parameter), in fact, $\varepsilon \rightarrow 0$, and $c(\varepsilon)$ a positive factor, normalizing the data in the space $\mathcal{L}^{2}\left(\mathbb{T}^{1}\right)$, i.e., on the period. Dr. D. Dix, my colleague at USC, conducted a series of computer experiments. I am deeply indebted to him for his work, and many useful discussions. Dix computed and plotted the $3 d$-graph of the density function $\rho=\rho\left(\vartheta_{\varepsilon}, t, x\right):=\left|\psi\left(\vartheta_{\varepsilon}, t, x\right)\right|^{2},(t, x) \in \mathbb{R}^{2}$, for $\varepsilon=0.01$, the contour map, and the so-called Bohm's trajectories.

Fig. 1 depicts "one quarter" (i.e., $(t, x) \in[0,1 / 2] \times[0,1 / 2])$ of the $3 d$-graph of $\rho$, and Fig. 2 is the contour (topographical) map, i.e., the level lines of the density $\rho$. Two features are apparent. First, the graphs represent a rugged "mountain landscape", and second, the landscape is not a completely random combination of "peaks and trenches". In particular, it is criss-crossed by a rather well-organized set of deep rectilinear canyons, or "the valleys of shadow" ${ }^{1}$. In Fig. 3, a family of so-called Bohm's trajectories can be seen (cf. [6]). In the

\footnotetext{
${ }^{1}$ Even though I walk through the valley of the shadow of death, I will fear no evil, for you are with me; your rod and your staff, they comfort me. Psalm 23 of David.
} 


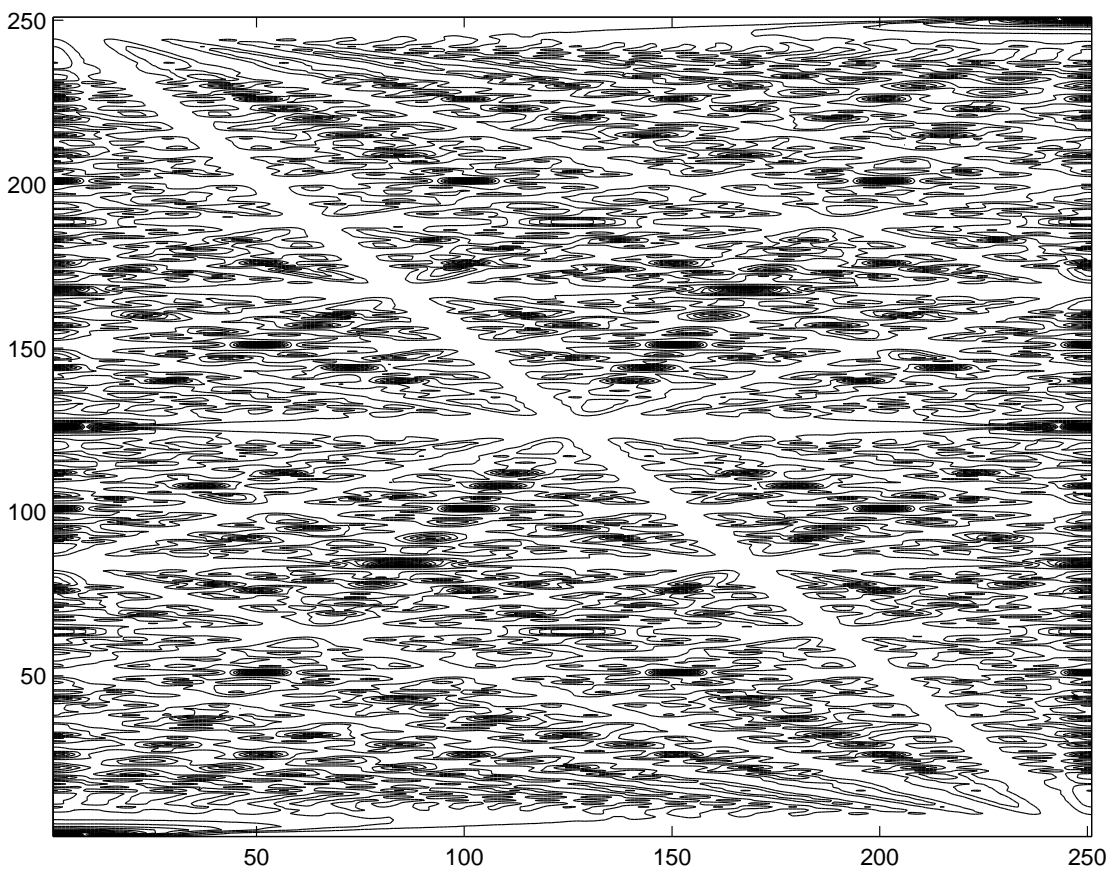

Fig. 2. Schrödinger density - the contour map

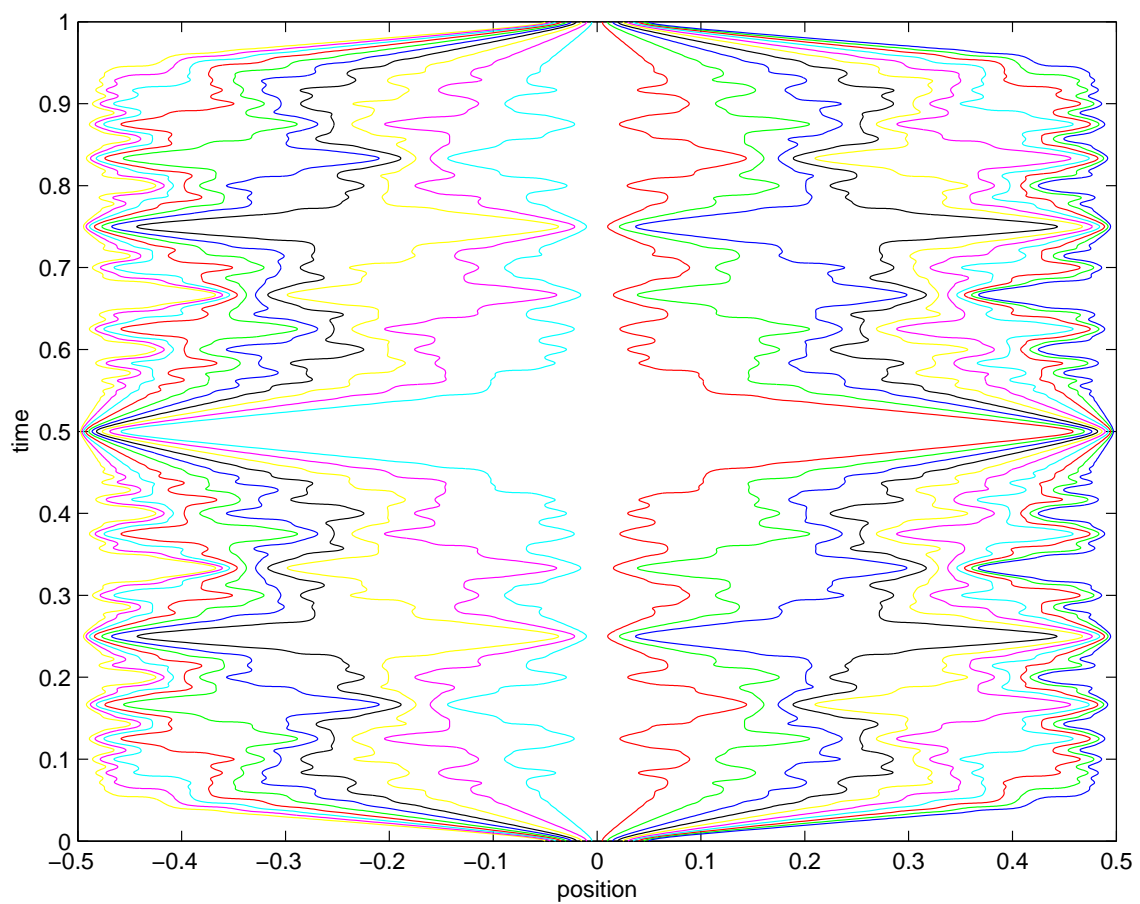

Fig. 3. Bohm's trajectories 
given case, the initial data is real-valued and positive, and these trajectories are curves, on which the solution $\psi$ conserves the initial value of the phase, i.e. remains real-valued and positive. Self-similarity, the main feature of fractals, is obvious on all three graphs.

Seeing this, the author decided to work out the answers to the following questions.

1) What is the description of "the valleys of shadow", and how "deep" are they?

2) What happens with the density function $\rho$ outside of the valleys?

3) Do these effects appear only as a result of the specific choice of the initial data, or they represent a typical property, a "seal" of the Schrödinger equation, for wide classes of compressed, narrowly supported data, or data functions with distinct singularities?

4) How does the introduction into the equation of a potential, generally neither smooth nor periodic, affect the self-similarity features of the solutions?

We denote

$$
\psi(f ; t, x):=\sum_{n \in \mathbb{Z}} \hat{f}_{n} e^{2 \pi i\left(n^{2} t+n x\right)}, \quad \rho(f ; t, x):=|\psi(f ; t, x)|^{2}
$$

the generalized solution, and, respectively, the arising density, for the Cauchy initial value problem

$$
\frac{\partial \psi}{\partial t}=\frac{1}{2 \pi i} \frac{\partial^{2} \psi}{\partial x^{2}},\left.\quad \psi(f ; t, x)\right|_{t=0}=f(x)=\sum_{n \in \mathbb{Z}} \hat{f}_{n} e^{2 \pi i n x} ;
$$

for $p \in[1, \infty], \mathcal{L}^{p}(\cdot)$ the usual notation for $\mathcal{L}^{p}$ function spaces on the specified subsets $(\cdot)$, with regard to the Lebesgue measure on these subsets; in the limiting case $p=\infty$, $\mathcal{L}^{\infty}$ denotes the space of essentially bounded functions with the norm ess $\sup |f| ; \mathcal{C}$ is the space of continuous functions with the norm $\max |f|$.

Let us introduce the notion of a periodic $\sqrt{\delta}$-family. The purpose of this notion is the following. The density $\rho$ considered in this paper is given by the squared modulus $|\psi|^{2}$ of the complex-valued solution $\psi$ of the problem (1), and for the initial density we have $\rho_{0}=\left.\rho(t, x)\right|_{t=0}=|f(x)|^{2}, x \in \mathbb{R}$, where $f$ is the initial data function of (1). The quantum-mechanical interpretation of the value $\rho(t, x)$, for a fixed time moment $t$ as a function of the variable $x$, is the probability density of locating the quantum particle at the point $x$. Thus, in the space-periodic case, for each fixed $t$ we have

$$
\int_{0}^{1} \rho(t, x) d x=\int_{0}^{1}|\psi(t, x)|^{2} d x=1
$$

which amounts to the assertion that the particle is somewhere on the period. The assumption that at the initial moment $t=0$ the particle is deterministic, i.e., located at a certain definite point on the period, say, at $x=0$, amounts therefore to saying that $\rho_{0}=|f|^{2}=\delta$ where $\delta$ denotes the periodic Dirac's delta function. Thus, for the problem (1) as a generator of physically meaningful densities $\rho$, of particular interest are the initial data $f$, which are $\mathcal{L}^{2}$-normalized on the period, i. e., in the space $\mathcal{L}^{2}\left(\mathbb{T}^{1}\right)$, and such that $|f|^{2}$ approximate $\delta$.

By the definition, a set of (generally, complex-valued) functions $\mathcal{F}=\left\{f_{\varepsilon}\right\}_{\varepsilon>0} \subset$ $\mathcal{L}^{2}\left(\mathbb{T}^{1}\right)$, parameterized by positive $\varepsilon$, is called a $\sqrt{\delta}$-family (root-delta family), if

$$
\forall g \in \mathcal{C}\left(\mathbb{T}^{1}\right): \quad \lim _{\varepsilon \rightarrow 0} \int_{0}^{1}\left|f_{\varepsilon}(x)\right|^{2} g(x) d x=g(0) .
$$


If, in addition, all functions of $\mathcal{F}$ are even, i.e. $f_{\varepsilon}(-x) \equiv f_{\varepsilon}(x)$, we say that $\mathcal{F}$ is an even root-delta family, and use the notation $\sqrt{\delta_{+}}$; if $f_{\varepsilon}(-x) \equiv-f_{\varepsilon}(x)$, the set $\mathcal{F}$ will be called an odd root-delta family, and the notation $\sqrt{\delta_{-}}$applied.

The family $\left\{\vartheta_{\varepsilon}\right\}$ of the Jacobi $\vartheta$-functions

$$
\vartheta_{\varepsilon}(x):=\sqrt[4]{\frac{2}{\varepsilon}} \sum_{m \in \mathbb{Z}} e^{-\frac{\pi(x-m)^{2}}{\varepsilon}}=\sqrt[4]{2 \varepsilon} \sum_{n \in \mathbb{Z}} e^{-\pi n^{2} \varepsilon} e^{2 \pi i n x}, \quad \varepsilon>0,
$$

suggested by Z. Ciesielski, is an example of a $\sqrt{\delta_{+}}$-family. Of course, there are many other classical $\sqrt{\delta}$-families, such as the Dirichlet kernels, wave packets, characteristic functions of intervals, and periodic Haar functions :

$$
w_{\varepsilon}(x):=\sqrt{\varepsilon} \sum_{n:|n| \varepsilon \leq 1 / 2} e^{2 \pi i n x}, \quad w_{\varepsilon, k}(x):=e^{2 \pi i k x} w_{\varepsilon}(x), k \in \mathbb{Z} ;
$$

$$
\tilde{\mathbf{1}}_{\varepsilon}(x):=\frac{1}{\sqrt{\varepsilon}} \sum_{m \in \mathbb{Z}} \mathbf{1}_{\varepsilon}(x-m) ; \quad \tilde{\chi}_{\varepsilon}(x):=\frac{1}{\sqrt{\varepsilon}} \sum_{m \in \mathbb{Z}} \chi_{\varepsilon}(x-m),
$$

where $\mathbf{1}_{\varepsilon}$ denotes the characteristic function of the interval $[-\varepsilon / 2, \varepsilon / 2]$, i.e. $\mathbf{1}_{\varepsilon}(x)=1$ if $|2 x| \leq \varepsilon$, and $\mathbf{1}_{\varepsilon}(x)=0$ for all other $x ; \chi_{\varepsilon}(x):=\mathbf{1}_{\varepsilon}(x) \operatorname{sign} x$. In physics literature, see e.g. [4],[5], [31], the initial data $\tilde{\mathbf{1}}_{\varepsilon}$ are known as Ronchi's grating (for $\varepsilon=1 / 2$ ). The initial densities $w_{\varepsilon}^{2}, \tilde{\mathbf{1}}_{\varepsilon}^{2}, \vartheta_{\varepsilon}^{2}$ are classical tools of the theory of trigonometric series, and approximation theory; in particular, $w_{\varepsilon}^{2}$ are known as Fejér's $(C, 1)$-summation kernels, see e.g. [40], v. 1 , Ch. $3 ; \tilde{\mathbf{1}}_{\varepsilon}^{2}$ is the kernel of the convolution operator of the moving average, or sliding window; $\vartheta_{\varepsilon}^{2}$ are the kernels of the Gauss summation method.

In the sequel, for fixed real numbers $N, \xi$, denote, respectively, $\mathbb{L}_{N, \xi}$ and $\mathbb{L}_{N, \xi}^{T}$, the following lines on the plane $\mathbb{R}^{2}$ :

$$
\mathbb{L}_{N, \xi}:=\mid\left\{\mathbf{x}=(t, x) \in \mathbb{R}^{2}: x+N t=\xi\right\}, \quad \mathbb{L}_{N, \xi}^{T}:=\left\{\mathbf{x}=(t, x) \in \mathbb{R}^{2}: N x+t=\xi\right\} .
$$

Given a line $\mathbb{L}$ on $\mathbb{R}^{2}$, denote $\left.\rho\right|_{\mathbb{L}}$ the restriction (trace) $)^{2}$ of the density $\rho$ on $\mathbb{L}$. For the initial data $f$ of the class $\mathcal{L}^{2}\left(\mathbb{T}^{1}\right),\left.\rho(f)\right|_{\mathbb{L}}$ is a (locally) integrable function on $\mathbb{L}$. This easily follows by termwise integration, over finite intervals belonging $\mathbb{L}$, of the double series that represents $\rho$. On a line $\mathbb{L}$ with a rational slope $N$, the corresponding trace $\left.\rho(f)\right|_{\mathbb{L}}$ is periodic.

Let $\mathcal{F}=\left\{f_{\varepsilon}\right\}_{\varepsilon>0}$ be a $\sqrt{\delta}$-family. We say that a (positive) density distribution $\rho_{\mathbb{L}}$ is the weak limit of $\left.\rho\left(f_{\varepsilon}\right)\right|_{\mathbb{L}}$ as $\varepsilon \rightarrow 0$, if for every continuous and compactly supported function $g$ on $\mathbb{L}$

$$
\left.\lim _{\varepsilon \rightarrow 0} \int_{\mathbb{L}} \rho\left(f_{\varepsilon}\right)\right|_{\mathbb{L}} g d \mu=\int_{\mathbb{L}} \rho_{\mathbb{L}} g d \mu ;
$$

$\mu$ denotes the usual Lebesgue measure on $\mathbb{L}$. If the limit $\rho_{\mathbb{L}}$ not only exists, but also is the same for all $\sqrt{\delta}$-families, we say that $\rho_{\mathbb{L}}$ is the trace of $\rho(\sqrt{\delta})$ on $\mathbb{L}$, and apply the notation $\rho(\sqrt{\delta} ; \mathbb{L}):=\rho_{\mathbb{L}}$. The definitions of the weak limits $\rho\left(\sqrt{\delta} ; \mathbb{R}^{2}\right), \rho\left(\sqrt{\delta_{+}} ; \mathbb{L}\right), \rho\left(\sqrt{\delta_{-}} ; \mathbb{L}\right)$

\footnotetext{
${ }^{2}$ The lack of space forces us to confine the understanding of the trace $\left.\rho\right|_{\mathbb{L}_{N, \xi}}$ as the univariate function resulting from the formal substitution $x=\xi-N t$ into the double Fourier series of $\rho$; the trace $\left.\rho\right|_{\mathbb{L}_{N, \xi}^{T}}$ is understood similarly. It seems interesting to clarify which conditions guarantee the existence of such traces in the distributional sense.
} 
are analogous, and the latter notations are applied if and only if the limits $\rho_{\mathbb{L}}$ exist, and do not depend on the $\sqrt{\delta}$-family, or, respectively, $\sqrt{\delta_{+}} \sqrt{\delta_{-}}$-families of the initial data.

Visually, the special lines on Fig. 1 and Fig. 2 are $\mathbb{L}_{N, \frac{M}{2}}$, where $N, M$ are integers. The following theorem contains an explanation, and answers a part of the raised questions concerning the limit densities.

Theorem 1. A) Assume that $N, M$ are integers. Then

$$
\rho\left(\sqrt{\delta_{+}} ; \mathbb{L}_{N, \frac{M}{2}}\right)=1+(-1)^{N M} ; \quad \rho\left(\sqrt{\delta_{-}} ; \mathbb{L}_{N, \frac{M}{2}}\right)=1-(-1)^{N M} .
$$

$B$ ) Assume that $N$ is a rational number, $N \notin \mathbb{Z}, \xi$-real, and $\tau$-irrational. Then

$$
\rho\left(\sqrt{\delta}, \mathbb{L}_{N, \xi}\right)=\rho\left(\sqrt{\delta}, \mathbb{L}_{0, \tau}^{T}\right)=\rho\left(\sqrt{\delta} ; \mathbb{R}^{2}\right)=1 .
$$

REMARK 1. Assume that $\tau$ is a rational number, $\tau=\frac{a}{q}, a \in \mathbb{Z}, q \in \mathbb{N}^{1},(a, q)=1$. Then

$$
\rho\left(\sqrt{\delta} ; \mathbb{L}_{0, \frac{a}{q}}^{T}\right)=\frac{1}{q} \sum_{m} \delta_{\frac{m}{q}} \begin{cases}1, & \text { if } q \equiv 1(\bmod 2), \\ 1+(-1)^{m+\frac{q}{2}}, & \text { if } q \equiv 0(\bmod 2) .\end{cases}
$$

REMARK 2. If $N, M$ are odd integers, and $\mathbb{L}=\mathbb{L}_{N, \frac{M}{2}}$, then according to $(3) \rho\left(\sqrt{\delta_{+}}, \mathbb{L}\right)=$ 0 , i.e. $\mathbb{L}$ is one of the "valleys of shadow". Since $\rho\left(f_{\varepsilon}\right)$ are positive functions, the weak limit equality $\rho\left(\sqrt{\delta_{+}}, \mathbb{L}\right)=0$ is equivalent to the statement of strong convergence in $\mathcal{L}_{\text {loc }}^{1}(\mathbb{L})$ : if $N$ is an odd integer, and $\mathcal{F}=\left\{f_{\varepsilon}\right\}_{\varepsilon>0}$ a $\sqrt{\delta_{+}}$-family, then

$$
\lim _{\varepsilon \rightarrow 0} \int_{0}^{1} \rho\left(f_{\varepsilon} ; t, N t+\frac{1}{2}\right)=0 .
$$

It can be seen in Fig. 2 that the density $\rho\left(\vartheta_{\varepsilon}\right)$ has a special character not only on the lines $\mathbb{L}_{N, \frac{M}{2}}$, but also on the transversal lines $\mathbb{L}_{N, \frac{M}{2}}^{T}$ where $M, N$ are odd integers. Although here the peculiarity is less apparent, and these lines are not "valleys of shadow", D. Dix expressed a conjecture, based on the numerical analysis and the graphics, that the density remains bounded as $\varepsilon \rightarrow 0$.

The next statement confirms this conjecture of Dix for the above mentioned classical $\sqrt{\delta_{+}}$-families of the initial data: $\vartheta$-functions, Dirichlet kernels, and Ronchi's gratings. (It does not seem likely, however, that the conjecture is true for general $\sqrt{\delta_{+}}$-families.)

TheOREM 2. Assume that $N, M$ are odd numbers, $\tilde{\mathbb{L}}:=\mathbb{L}_{N, \frac{M}{2}} \cup \mathbb{L}_{N, \frac{M}{2}}^{T}$, and the family $\mathcal{F}=\left\{f_{\varepsilon}\right\}_{\varepsilon>0}$ consists of the functions $\vartheta_{\varepsilon}, w_{\varepsilon}$ or $\mathbf{1}_{\varepsilon}$, see (2). Then

$$
\sup _{\varepsilon \in(0,1)}\left\|\rho\left(f_{\varepsilon}\right)\right\|_{\mathcal{L}^{\infty}(\tilde{\mathbb{L}})}<\infty .
$$

For these initial data, the densities $\rho\left(f_{\varepsilon}\right)$ tend to 0 on the line $\mathbb{L}_{N, \frac{M}{2}}$ not only in $\mathcal{L}^{1}$, but also in $\mathcal{L}^{p}$ for all $p<\infty$.

REMARK 3. The lines $\mathbb{L}_{N, \frac{M}{2}}, \mathbb{L}_{N, \frac{M}{2}}^{T}$ with odd $N, M$, where the density remains bounded, are exceptional, indeed. Thus, it can be proved that the trace of the density $\rho\left(w_{\varepsilon}\right)$, as $\varepsilon \rightarrow 0$, is almost everywhere unbounded on each of the lines $\mathbb{L}_{N, M}, \mathbb{L}_{N, M}^{T}$ with integer $N, M$, and for almost all $\xi$

$$
\sup _{\varepsilon \in(0,1)}\left\|\rho\left(w_{\varepsilon}\right)\right\|_{\mathcal{L}^{\infty}\left(\mathbb{L}_{0, \xi}\right)}=\left\|\rho\left(w_{\varepsilon}\right)\right\|_{\mathcal{L}^{\infty}\left(\mathbb{L}_{0, \xi}^{T}\right)}=\infty .
$$


Theorem 2 is deeper than theorem 1. The proof requires the local estimates of the elliptic $\vartheta$-function of complex argument $\vartheta(z):=\sum_{n \in \mathbb{Z}} e^{-n^{2} z}, \quad \Re z>0$, the estimates of the exponential sums with the quadratic phase $\sum_{\nu=1}^{n} e^{2 \pi i\left(\nu^{2} t+\nu x\right)}$, and the results concerning the local properties of the discrete oscillatory Hilbert transforms, see section 8 below,

$$
H(t, x):=\text { p.v. } \sum_{n \in \mathbb{Z} \backslash\{0\}} \frac{e^{2 \pi i\left(n^{2} t+n x\right)}}{2 \pi i n} .
$$

In the next few sections, we provide some comments on the fractal nature of the solutions of a wide class of Schrödinger type equations with the periodic initial data. We also discuss the relations with the optical effect of W. H. F. Talbot [35]. The proofs of the theorems are contained in sections $6-8$.

2. $V$-extensions. In the earlier papers [25]-[27] (a review can be found in [28]), the author studied the properties of the solutions $\psi$, and also of an essentially wider class of trigonometric series, called Vinogradov's extensions, for brevity, $V$-extensions.

Vinogradov's extensions appear quite naturally, via the classical method of Fourier of separation of variables, in the study of the properties of the solutions of the Cauchy initial value problem posed for a wide variety of Schrödinger type equations. The latter include not just the problem (1) for a "free quantum particle in a box", but, for example, the linearized version of the Korteweg-deVries equation (KdV). We briefly discuss this subject in the subsequent section 08; for more details and references, see [28]. Let us only notice here, that the results and the methods of analytic number theory, in particular, the circle method of Hardy-Littlewood-Ramanujan-Vinogradov, are crucial components of the study, if the initial data function is periodic, and the goal is understanding of the pointwise properties of the solutions. The latter especially concerns non-trivial situations, when the series do not converge absolutely. For example, the circle method is a crucial component of the proof of global boundedness of the solution of the linearized $\mathrm{KdV}$ equation for the initial data that are periodic functions of bounded variation on the period (i.e., of the class $B V\left(\mathbb{T}^{1}\right)$ ).

In general, the case of periodic initial data is complicated, in comparison with the non-periodic case, by the necessity of the application of the micro-local analysis, see e.g. [19]. However, it does not mean that the non-periodic case is easy. Here, the technics and methods are based on the analysis of the oscillatory integrals. A pioneering work in the direction of the regularity conditions of the solutions of the Schrödinger equation with the non-periodic (compactly supported) initial data is due to L. Carleson [8], which has later found multiple developments, see e.g. [10], [21], [32].

A $V$-extension (of degree $r \in \mathbb{N}, r \geq 2$ ) of a periodic function $f$ is defined as the sum, wherever it exists, of the oscillatory series with the real algebraic polynomial phase of higher degree in the exponentials:

$$
V(f ; \mathbf{x}):=\sum_{n \in \mathbb{Z}} \hat{f}_{n} e^{2 \pi i P(\mathbf{x}, n)}, \quad P(\mathbf{x}, n):=x_{r} n^{r}+\cdots+x_{1} n, \quad \mathbf{x}=\left(x_{r}, \ldots, x_{1}\right) \in \mathbb{R}^{r} .
$$

Vinogradov's extensions, and in particular, the solutions of the Cauchy problem (1), are self-similar. Self-similarity is the most typical feature of a $V$-extension, and the clas- 
sical complete rational exponential sums (Gauss sums of higher order) play the role of scaling factors.

Rather superficially, the source of self-similarity can be seen if we consider $V$-extensions for fixed rational values of the "senior" coefficients of the polynomial in the exponent $x_{r}, \ldots, x_{2}$, as a function of the variable "junior" coefficient $x=x_{1}$. In the sequel, $\mathbb{Q}^{r}$ denotes the following subset of the rational points in $\mathbb{R}^{r}$ :

$$
\mathbb{Q}^{r}:=\left\{\mathbf{y}=\left(y_{r}, \ldots, y_{2}, y_{1}\right)=\left(\frac{a_{r}}{q_{r}}, \cdots, \frac{a_{2}}{q_{2}}, \frac{m}{q}\right), q:=\left[q_{r}, \ldots, q_{2}\right], m \in \mathbb{Z}\right\}
$$

where $q_{s} \in \mathbb{N}^{1}, a_{s} \in \mathbb{Z},\left(a_{s}, q_{s}\right)=1, s=r, \ldots, 2$, and $q=q(\mathbf{y}):=\left[q_{r}, \ldots, q_{2}\right]$ is the least common multiple of the denominators of the rational numbers $y_{r}, \ldots, y_{2}$.

We have

$$
V\left(f ; \frac{a_{r}}{q_{r}}, \cdots, \frac{a_{2}}{q_{2}}, x\right)=\sum_{n \in \mathbb{Z}} \hat{f}_{n} e^{\frac{2 \pi i P(n)}{q}} e^{2 \pi i n x}, \quad P(n):=q\left(\frac{a_{r} n^{r}}{q_{r}}+\cdots+\frac{a_{2} n^{2}}{q_{2}}\right) .
$$

It follows that $V$ as a function of $x$, appears as a result of the multiplier transformation of the Fourier series of the initial data $f$. The multiplier $\left\{e^{\frac{2 \pi i P(n)}{q}}\right\}_{n \in \mathbb{Z}}$ is a periodic function of $n \in \mathbb{Z}$, of period $q$. Consequently, applying the discrete Fourier transform, we see that

$$
\begin{aligned}
& V\left(f ; \frac{a_{r}}{q_{r}}, \cdots, \frac{a_{2}}{q_{2}}, x\right)=\sum_{m=1}^{q} G\left(\mathbf{y}^{(m)}\right) f\left(x-\frac{m}{q}\right), \\
& \mathbf{y}^{(m)}:=\left(\frac{a_{r}}{q_{r}}, \cdots, \frac{a_{2}}{q_{2}}, \frac{m}{q}\right), \quad G\left(\mathbf{y}^{(m)}\right)=\frac{1}{q} \sum_{n=1}^{q} e^{2 \pi i \frac{P(n)+m n}{q}} .
\end{aligned}
$$

Therefore, in the given context, $V$ is a $q$-term linear combination of the translates of the initial data $f$.

The coefficients $G$ in these linear combinations are universal, they do not depend from the initial data. They are (normalized) complete rational exponential sums, or Gauss sums of higher order. The following identities ("conservation laws") are elementary:

$$
\sum_{m=1}^{q} G\left(\mathbf{y}^{(m)}\right)=\sum_{m=1}^{q}\left|G\left(\mathbf{y}^{(m)}\right)\right|^{2}=1 .
$$

In the particular case of $r=2$, the coefficients $G$ are the classical quadratic Gauss sums,

$$
G\left(\frac{a}{q}, \frac{m}{q}\right)=\frac{1}{q} \sum_{n=1}^{q} e^{2 \pi i \frac{a n^{2}+m n}{q}}, \quad(a, q)=1
$$

and the magnitudes of these complex numbers are well-known, see also (4),

$$
\left|G\left(\frac{a}{q}, \frac{m}{q}\right)\right|^{2}=\frac{1}{q} \begin{cases}1, & \text { if } q \equiv 1(\bmod 2), \\ 1+(-1)^{m+\frac{q}{2}} & \text { if } q \equiv 1(\bmod 2) .\end{cases}
$$

Therefore, if $q$ is odd, the solution operator $\psi\left(f ; \frac{a}{q}, x\right)=V\left(f ; \frac{a}{q}, x\right)$ of the problem (1)reproduces $q$ copies on the torus $\mathbb{T}^{1}$, of the initial function $f$, while if $q$ is even, the number of such (non-zero) copies is $\frac{q}{2}$.

Of course, the term "copy" is only conditionally applicable in this interpretation of (6), because different translates of the initial data $f\left(x-\frac{m}{q}\right)$ generally interfere with each 
other. However, if the function $f(x)$ is a "sharp image", say, it is supported in a narrow neighborhood $|x| \leq \varepsilon$ of the origin (like Ronchi's grating $\mathbf{1}_{\varepsilon}$, cf. (2)), and if the rational moment of time $t=\frac{a}{q}$ is such that $q \varepsilon \leq 1$, then the solution operator $\psi$ is a "copy machine", indeed, because the supports of the translates do not overlap. On the other hand, if $q \varepsilon>2$, the interference between translates of $f$ blends the sharp separate copies unrecognizably.

The same applies for higher degree $V$-extensions. Summarizing, we see that the discrete functions (distributions) on $\mathbb{R}^{r}$

$$
\mathcal{G}:=\sum_{\mathbf{y} \in \mathbb{Q}^{r}} G(\mathbf{y}) \delta_{\mathbf{y}}, \quad \mathcal{G}^{2}:=\sum_{\mathbf{y} \in \mathbb{Q}^{r}}|G(\mathbf{y})|^{2} \delta_{\mathbf{y}},
$$

of the arithmetical comb type, are intrinsically associated with the general $V$-extensions of periodic initial data, and consequently, with the solutions of a wide class of Schrödinger type equations. However, a big difference with the generic case $r=2$ is that the rational sums $G(\mathbf{y})$ of degree $r \geq 3$ are significantly more complicated arithmetical functions, than the quadratic Gauss sums. Anyhow, the set of rational points $\mathbf{y}$ for which $G(\mathbf{y}) \neq 0$ is everywhere dense in $\mathbb{R}^{r}$, so that a general $V$-extension $V(f, \mathbf{x})$ is a self-similar function, indeed. In particular, the sum of the series with the cubic phase

$$
u(f ; t, x)=\sum_{n \in \mathbb{Z}} \hat{f}_{n} e^{2 \pi i\left(n^{3} t+n x\right)},
$$

is self-similar. This series represents the solution of the Cauchy initial value problem for the linearized Korteweg-deVries equation with the periodic initial data function

$$
\frac{\partial u}{\partial t}=-\frac{1}{4 \pi^{2}} \frac{\partial^{3} u}{\partial x^{3}},\left.\quad u(t, x)\right|_{t=0}=f(x)=\sum_{n \in \mathbb{Z}} \hat{f}_{n} e^{2 \pi i n x},
$$

and in this case, the scaling factors are complete cubic rational sums

$$
G\left(\frac{a}{q}, 0, \frac{m}{q}\right)=\frac{1}{q} \sum_{n=1}^{q} \cos 2 \pi\left(\frac{a n^{3}+m n}{q}\right) .
$$

In this relation, curious examples of self-similar functions are provided by the sums of the following sine-series with the cubic phase:

$$
A(t):=\sum_{n=1}^{\infty} \frac{\sin 2 \pi n^{3} t}{\pi n}, \quad B(t, x):=\sum_{n=1}^{\infty} \frac{\sin 2 \pi\left(n^{3} t+n x\right)}{\pi n} .
$$

Both series converge everywhere, and the partial sums are uniformly bounded, according to a more general theorem from [2], see also section 8 . The function $B$ represents the solution $u(f ; t, x)$ of the problem (9) with the initial data $f(x):=\frac{1}{2}-\{x\}$, where $\{\cdot\}$ denotes the fractional part function. Thus, $A(t)$ describes the time-evolution, in accordance with (9), of one single "unit jump" on the period in the space variable. This jump is reproduced on an everywhere dense set of the rational $t=\frac{a}{q}$, where $A$ has discontinuities of the first kind (jumps). The value of the "little jump" at $t=\frac{a}{q}$ equals the cubic rational sum $G\left(\frac{a}{q}, 0,0\right)$; for more details, the reader may be referred to the earlier review paper [28].

REMARK 4. For $r=2$, the zeros of the Gauss arithmetic function $G(\mathbf{y})$ align themselves along the "valleys of shadow", i.e. on the lines $\mathbb{L}_{N, \frac{M}{2}}$ with odd $N, M$, see also lemma 1 
below. It would be interesting to clarify whether or not the zeros of $G(\mathbf{y})$ for $r \geq 3$ also align themselves along manifolds in $\mathbb{R}^{r}$ that can be described in geometrically transparent terms.

3. The circle method and self-similarity. A much deeper fact is the self-similarity in H. Weyl's exponential sums

$$
W_{n}(\mathbf{x}):=\frac{1}{n} \sum_{\nu=1}^{n} e^{2 \pi i P(\mathbf{x}, \nu)}, \quad \mathbf{x}:=\left(x_{r}, \ldots, x_{1}\right) \in \mathbb{R}^{r}, n \in \mathbb{N},
$$

when these sums are considered globally, as functions of $r+1$ variables: the natural $n$, and the real coefficients $x_{r}, \ldots, x_{1}$ of the algebraic polynomial in the exponent. This property has been well understood, and utilized, in analytic number theory since the beginning of the 20th century, when the famous circle method of Hardy-Littlewood-RamanujanVinogradov was created. This method combines estimates and asymptotic formulas for Weyl's sums. The original goal was Waring's problem, however, the scope of applications of the circle method in number theory alone is immense.

As an illustration of the self-similar properties in Weyl's sums, let us consider the asymptotic formulas on the so-called major arcs. G. I. Arkhipov [1] established the following variant of Vinogradov's [37] method of exponential sums. Given a (large) natural number $n$, denote by $\square_{n}$ the "rectangular box"

$$
\square_{n}^{r}:=\left\{\mathbf{z}=\left(z_{r}, \ldots, z_{1}\right) \in \mathbb{R}^{r},\left|z_{s}\right| \leq n^{0.3-s}, s=r, \ldots, 1\right\},
$$

and consider the subset $\mathbb{Q}_{n}^{r}$ of the rational points in $\mathbb{R}^{r}$ with "relatively small denominators" (see also the previous section)

$$
\mathbb{Q}_{n}^{r}:=\left\{\mathbf{y}=\left(\frac{a_{r}}{q_{r}}, \cdots, \frac{a_{2}}{q_{2}}, \frac{a_{1}}{q}\right), q=q(\mathbf{y}):=\left[q_{r}, \ldots, q_{2}\right] \leq n^{0.3}\right\} .
$$

The major arc $A_{n}$ is defined as the union of the boxes, centered at the points of $\mathbb{Q}_{n}^{r}$ : $A_{n}=Q_{n}^{r}+\square_{n}^{r}$; for $\mathbf{y} \in \mathbb{Q}_{n}^{r}, \mathbf{z} \in \square_{n}^{r}$ the following asymptotic formulas are valid:

$$
\begin{aligned}
& \text { (i) } W_{n}(\mathbf{z})=\mathcal{W}_{n}(\mathbf{z})+O\left(n^{-0.7}\right), \quad \mathcal{W}_{n}(\mathbf{z}):=\frac{1}{n} \int_{0}^{n} e^{2 \pi i P(\mathbf{z}, \nu)} d \nu ; \\
& \text { (ii) } W_{n}(\mathbf{y}+\mathbf{z})=G(\mathbf{y}) \mathcal{W}_{n}(\mathbf{z})+O\left(n^{-0.7}\right) .
\end{aligned}
$$

The complete rational sums $G(\mathbf{y})$ appear as scaling factors in these formulas, while the "pattern" is given by the continuous analog $\mathcal{W}_{n}$ of the sum $W_{n}$, i.e. the oscillatory integral with the algebraic polynomial phase. A useful estimate of the integral in many applications, in particular, in Schrödinger type equations, is the following:

$$
\left|\mathcal{W}_{n}(\mathbf{z})\right| \leq \min \left(1, \frac{c}{P_{*}^{1 / r}(\mathbf{z}, n)}\right), \quad P_{*}(\mathbf{z}, n):=\left|z_{r}\right| n^{r}+\cdots+\left|z_{1}\right| n .
$$

This estimate is an equivalent form of Vinogradov's lemma [37], Ch. 2, on oscillatory integrals, cf. also [24].

As for the minor arc, which by the definition is the complement of the major arc, $B_{n}:=\mathbb{R}^{r} \backslash A_{n}$, Weyl's sums satisfy here the estimate

$$
W_{n}(\mathbf{x})=O\left(n^{-\alpha}\right), \quad \alpha=\left(8 r^{2}(\ln r+1,5 \ln \ln r+4.2)\right)^{-1}, \quad \mathbf{x} \in B_{n}, n \rightarrow \infty .
$$


4. An excerpt from the work of W. H. F. Talbot In optics, the multi-scaled selfsimilarity effects were experimentally discovered as early as in 1836 by W. H. F. Talbot [35], the British inventor of photography.

Below is an excerpt from this wonderful publication of Talbot.

... In order to see these appearances in their perfection, it is requisite to have a dark chamber and a radiant point of intense solar light, which, for the sake of convenience, should be reflected horizontally by a mirror. I will relate a few, out of several experiments which were made in this manner.

1. About ten or twenty feet from the radiant point, I placed in the path of the ray an equidistant grating (a plate of glass covered with gold-leaf, on which several hundred parallel lines are cut, in order to transmit the light at equal intervals) made by Fraunhofer, with its lines vertical. I then viewed the light which had passed through the grating with a lens of considerable magnifying power. The appearance was very curious, being a regular alternation of numerous lines of red and green colour, having their direction parallel to the lines of the grating. On removing the lens a little further from the grating, the bands gradually changed their colours, and became alternately blue and yellow. When the lens was a little more removed, the bands again became red and green. And this change continued to take place for an indefinite number of times, as the distance between the lens and the grating increased. In all cases the bands exhibited two complementary colours.

It was very curious to observe that though the grating was greatly out of focus of the lens, yet the appearance of the bands was perfectly distinct and well defined.

This however only happens when the radiant point has a very small apparent diameter, in which case the distance of the lens may be increased even to one or two feet from the grating without much impairing the beauty and distinctness of the coloured bands. So that if the source of light were a mere mathematical point it appears that this distance might be increased without limit; or that the disturbance of the luminous indulations caused by the interposition of the grating, continues indefinitely, and has no tendency to subside of itself.

2. Another grating was then placed at right angles to the first, and the light transmitted through both was examined by the lens. The appearance now resembled a tissue woven with red and green threads. It seemed exactly as if each colour disappeared alternately behind the other. An alteration in the distance of the lens, altered the two complementary colours.

3. A plate of copper pierced with small circular holes of equal diameter and in regular rows, was substituted for the gratings. When this plate was held perpendicular to the ray, it produced a beautiful pattern consisting of rows of circles divided by coloured lines or bars. When the lens was approached to the plate, there was a particular distance between them at which it appeared in the centre of each circle a black spot, as small and well defined in appearance as a full point in a printed book, being a curious instance of the well-known fact, of the interference of rays of light producing darkness. This black spot was seen in all circles at once, in consequence of their having equal diameters.

4. When the copper-plate was placed obliquely and held in various positions, a great variety of very singular patterns were dispayed, which can be compared to nothing so well as to tissues woven with threads of various colours. It would be impossible to describe these, and more than the ever-changing figures of the kaleidoscope. They seem to vary ad infinitum, and in whatever position the plate is placed, they appear as distinct as if they were in the focus of the lens...

The author of the present paper learnt about Talbot's phenomenon from the manuscript of the work [22] which he happened to referee, and from the subsequent acquaintance with the physics literature, e.g. [3]-[5], [31]. The modern physics terminology with regard to the self-similarity features includes quantum carpets, self-imaging; integer, fractional and fractal revivals, etc. The integer revival reflects that the solution of the Cauchy 
problem (1) with the space-periodic initial data is periodic in both space and time variables, $\psi(f ; t+1 / 2, x+1 / 2) \equiv \psi(f ; t, x) \equiv \psi(f ; t+1, x) \equiv \psi(f ; t, x+1)$. Self-imaging reflects the property of the multi-scaled reproduction of the initial data. Fractional and fractal revivals mean that at rational moments of time $t=\frac{a}{q}$ with small denominators $q$, the copies of the initial data reappear in the sharp focus from a blended mass. This group of phenomena is illustrated above by the relation (6) for general Vinogradov's extensions. A typical quantum carpet is depicted in Fig. 2, and the functions $\mathcal{G}, \mathcal{G}^{2}$, see (8), are quantum combs.

5. Equations: Wave $\Rightarrow$ Helmholtz $\Rightarrow$ Schrödinger. Paraxial approximation. It seems proper to provide a partial argument, mathematically not meticulous, why the fractal properties of the Schrödinger wave function $\psi$ could so brightly manifest themselves in the optics phenomenon discovered by Talbot. We borrow the explanation from the papers [4], [22], with some minor modifications, and again emphasize, that from the mathematical point of view, the arguments below are by far not sufficiently justified. The latter remark especially concerns the so-called paraxial approximation, see "Ansatz 3" below. An elaboration of a deeper "mathematization" of these arguments seems to be of considerable interest to the author.

The assumption is that the diffracted light wave in the space past the grating satisfies the wave equation

$$
\left(\frac{1}{c^{2}} \frac{\partial^{2}}{\partial t^{2}}-\Delta\right) W=0, \quad \Delta:=\frac{\partial^{2}}{\partial x^{2}}+\frac{\partial^{2}}{\partial y^{2}}+\frac{\partial^{2}}{\partial z^{2}}
$$

where $c$ denotes the speed of light in vacuum, $t$ - the time variable; $(\mathbf{x}, z):=(x, y, z)$ is the Cartesian coordinate system erected so that $z=0$ describes the "grated" plane $\mathbb{R}^{2}=\{\mathbf{x}=(x, y)\}$, and the half-space past the plane is given by $z>0$. The light is assumed to be monochromatic, of some fixed frequency $\omega .{ }^{3}$ Accordingly, the solution of the wave equation is assumed to have the form $W=e^{2 \pi i \omega t} U(\mathbf{x}, z)$ where $U$ is the complex local amplitude. The boundary value $U(\mathbf{x}, 0)=f(\mathbf{x}), \mathbf{x}=(x, y) \in \mathbb{R}^{2}$, models the original image on the diffraction plane, so that the mathematical "Ansatz 1" of the problem is

$$
\begin{gathered}
W(t, \mathbf{x}, z)=e^{2 \pi i \omega t} U(\mathbf{x}, z) ; \quad\left(\Delta+\left(\frac{2 \pi}{\lambda}\right)^{2}\right) U=0 \\
\left.U(\mathbf{x}, z)\right|_{z=0}=f(\mathbf{x}), \quad \mathbf{x}=(x, y) \in \mathbb{R}^{2}
\end{gathered}
$$

where $\lambda:=\frac{c}{\omega}$ denotes the wave-length of the light signal. Concerning $U$, one needs to solve the boundary value problem for Helmholtz equation. The Fourier method of separation of variables is applied for this purpose:

$U(\mathbf{x}, z)=\int_{\mathbb{R}^{2}} \hat{f}(\mathbf{y}) e^{2 \pi i(\Omega(\mathbf{y}) z+\mathbf{x} \cdot \mathbf{y})} d \mathbf{y}, \quad \hat{f}(\mathbf{y})=\int_{\mathbb{R}^{2}} f(\mathbf{x}) e^{-2 \pi i \mathbf{y} \cdot \mathbf{x}} d \mathbf{x}, \quad \mathbf{y}=\left(y_{1}, y_{2}\right) \in \mathbb{R}^{2}$.

\footnotetext{
${ }^{3}$ The given explanation seemingly does not apply to the effects of bands changing colors in the experiment of Talbot, who used a radiant point of intense solar light as a source, i.e. white light, the composition of several frequencies.
} 
Since for a fixed vector $\mathbf{y} \in \mathbb{R}^{2}$

$$
\left(\Delta+\left(\frac{2 \pi}{\lambda}\right)^{2}\right) e^{2 \pi i(\Omega z+\mathbf{x} \cdot \mathbf{y})}=4 \pi^{2}\left(\left(\lambda^{-2}-|\mathbf{y}|^{2}\right)-\Omega^{2}\right) e^{2 \pi i(\Omega z+\mathbf{x} \cdot \mathbf{y})},
$$

the basic equation for the selection of $\Omega=\Omega(y)$, according to the Fourier method, is

$$
\Omega^{2}(y)=\lambda^{-2}-|\mathbf{y}|^{2} .
$$

Of course, this equation is not enough for a single-valued determination of $\Omega(y)$, and here is "Ansatz 2" made in [4],[22]: physics of the problem suggests the following solution

$$
\begin{aligned}
& \Omega(y):=\sqrt{\lambda^{-2}-|\mathbf{y}|^{2}}=\frac{\sqrt{1-|\lambda \mathbf{y}|^{2}}}{\lambda}, \text { for }|\mathbf{y}| \leq \lambda^{-1} ; \\
& \Omega(y):=i \frac{\sqrt{|\lambda \mathbf{y}|^{2}-1}}{\lambda}, \text { for }|\mathbf{y}|>\lambda^{-1},
\end{aligned}
$$

so that the eigen-functions "shall not explode" for large values of $|\mathbf{y}|, z$, and on the contrary, decay exponentially.

"Ansatz 3" consists in the substitution of $U(\mathbf{x}, z)$ by its paraxial approximation

$$
U(\mathbf{x}, z) \approx \tilde{U}(\mathbf{x}, z):=e^{2 \pi i \frac{z}{\lambda}} \int_{\mathbb{R}^{2}} \hat{f}(\mathbf{y}) e^{\pi i\left(-|y|^{2} \lambda z+2 \mathbf{x} \cdot \mathbf{y}\right)} d \mathbf{y} .
$$

The idea of this approximation is that only the low frequency domain $\lambda|\mathbf{y}| \ll 1$ provides a significant input,

$$
\int_{\mathbb{R}^{2}} \hat{f}(\mathbf{y}) e^{2 \pi i(\Omega(\mathbf{y}) z+\mathbf{x} \cdot \mathbf{y})} d \mathbf{y} \approx \int_{\lambda|\mathbf{y}| \ll 1} \hat{f}(\mathbf{y}) e^{2 \pi i(\Omega(\mathbf{y}) z+\mathbf{x} \cdot \mathbf{y})} d \mathbf{y},
$$

and in this domain $\sqrt{1-|\lambda \mathbf{y}|^{2}}$ approximately equals its two-term Taylor expansion, so that

$$
\Omega(\mathbf{y}) \approx \frac{1}{\lambda}\left(1-\frac{\lambda^{2}|\mathbf{y}|^{2}}{2}\right)
$$

Obviously,

$$
\int_{\mathbb{R}^{2}} \hat{f}(\mathbf{y}) e^{\pi i\left(-|y|^{2} \lambda z+2 \mathbf{x} \cdot \mathbf{y}\right)} d \mathbf{y}=\psi\left(f ;-\frac{\lambda z}{2}, \mathbf{x}\right),
$$

so that the result is the paraxial approximation of the solution of the original problem for wave equation by the solution of the Cauchy initial value problem for the Schrödinger equation:

$$
W(t, \mathbf{x}, z) \approx e^{2 \pi i \omega\left(t+\frac{z}{c}\right)} \psi\left(f ;-\frac{\lambda z}{2}, \mathbf{x}\right) .
$$

The three optical experiments described by Talbot correspond, respectively, to the following three classes of periodic initial data functions:
(I) $f(x, y)=f(x)$;
(II) $f(x, y)=f(x)+f(y)$;
(III) $f(x, y)=f(x) f(y)$,

where $f(x)=\sum_{n \in \mathbb{Z}} \hat{f}_{n} e^{\frac{2 \pi i n x}{\Lambda}}, \quad x \in \mathbb{R}^{1}$, 
and $\Lambda$ denotes the period of grating. The respective $\psi$-functions are given by

$$
\begin{aligned}
& \text { (I) } \psi_{1}(z, x)=\sum_{n \in \mathbb{Z}} \hat{f}_{n} e^{-\frac{\pi i z \lambda n^{2}}{\Lambda^{2}}} e^{\frac{2 \pi i n x}{\Lambda}} ; \quad \text { (II) } \psi_{2}(z, x, y)=\psi_{1}(z, x)+\psi_{1}(z, y) ; \\
& \text { (III) } \psi_{3}(z, x, y)=\psi_{1}(z, x) \psi_{1}(z, y) .
\end{aligned}
$$

The number $z_{T}:=\frac{2 \Lambda^{2}}{\lambda}$ equals the period, in the $z$-variable, of all three $\psi$-functions, it is known in optics as Talbot's distance. The lengths $\lambda$ of waves of the visible light are contained in the interval $\lambda \in[4.55,6.50] 10^{-5} \mathrm{~cm}$, while the realistic grating period $\Lambda$ in Talbot's experiment presumably lay in the interval $\Lambda \in\left[10^{-2}, 10^{-1}\right] \mathrm{cm}$. Therefore, realistic values of Talbot's distance in optical experiments are contained in the interval from decimeters to tens of meters.

The restriction $\lambda|\mathbf{y}|=\frac{\lambda|n|}{\Lambda} \ll 1$ determines the spectral interval of adequate representation of the true solution $U$ of the Helmholtz equation by its paraxial approximation $\tilde{U}$. This means, that either the initial data $f$ has to be a trigonometric polynomial of degree $\ll \frac{\Lambda}{\lambda}$, or a "sufficiently smooth" function that can be approximated by such polynomials with a small error. The latter is an evidence in favor of "mathematical reliability" of such approximation: the ratio $\frac{\Lambda}{\lambda}$ is sufficiently big, of orders $10^{3}-10^{4}$. The subspaces of trigonometric polynomials of such high orders are sufficiently rich to support the phenomena both in physics, and the computational experiments.

REMARK 4. In the half-space $\mathbb{R}_{+}^{3}:=\{(x, y, z), z>0\}$ past Talbot's grating plane, let us introduce the normalized co-ordinates $\left.\xi=\frac{x}{\Lambda}, \eta=\frac{y}{\Lambda}, \zeta=\frac{z}{z_{T}}\right)$. According to the representations (14), and theorems 1 and 2, one could expect that the light intensity in Talbot's experiments exhibits special features (of being everywhere bounded, or even small) on the following set of domains $\left(\left(N_{k}, M_{k}\right)\right.$ are arbitrary pairs of odd integers):

(I) in the first experiment, on the hyperplanes $\xi+N \zeta=\frac{M}{2}$ and $\zeta+N \xi=\frac{M}{2}$,

(II, III) in the second, and the third experiments, on the lines

$$
\begin{aligned}
& \frac{\xi-M_{1} / 2}{N_{1}}=\frac{\eta-M_{2} / 2}{N_{2}}=\zeta ; \quad N_{1} \xi-\frac{M_{1}}{2}=N_{2} \eta-\frac{M_{2}}{2}=\zeta ; \\
& \frac{\xi-M_{1} / 2}{N_{1}}=N_{2} \eta-\frac{M_{2}}{2}=\zeta ; \quad \frac{\eta-M_{1} / 2}{N_{1}}=N_{2} \xi-\frac{M_{2}}{2}=\zeta .
\end{aligned}
$$

It may be interesting to test whether or not these theoretical conclusions can be confirmed by experiments.

\section{Arithmetics of the density. The proof of Theorem 1. Wigner's functions.} The next statement provides an "arithmetical explanation" of the effect of the valleys of shadow in the Schrödinger landscape. The operator $\psi$ "fails to copy" the initial data on $\mathbb{L}_{N, \frac{M}{2}}$ and $\mathbb{L}_{N, \frac{M}{2}}^{T}$ with odd $M, N$, because the Gauss sums vanish on these lines. However, for $N \neq \pm 1$, the behavior of the density on these two types of lines is different. On each $\mathbb{L}_{N, \frac{M}{2}}$, the average density is small for the initial data of all $\sqrt{\delta+}$-families, while it is not small on $\mathbb{L}_{N, \frac{M}{2}}^{T}$. Here the density converges to 1 in the sense of distributions, see theorem 1 .

Lemma 1. Denote $\mathcal{G}:=\left\{\mathbf{y} \in \mathbb{Q}^{2}, G(\mathbf{y})=0\right\}$ the set of zeros of the complete Gauss sums, see also (7); $\mathbb{V}$, and, respectively, $\mathbb{V}^{T}$ the unions of the "valleys of the shadows", and the 
transversal lines on the plane $\mathbb{R}^{2}$ :

$$
\mathbb{V}:=\bigcup_{N, M \equiv 1(\bmod 2)} \mathbb{L}_{N, \frac{M}{2}}, \quad \mathbb{V}^{T}:=\bigcup_{N, M \equiv 1(\bmod 2)} \mathbb{L}_{N, \frac{M}{2}}^{T} .
$$

Then

$$
\text { a) } \mathcal{G}=\mathbb{Q}^{2} \cap \mathbb{V} ; \quad \text { b) } \mathbb{Q}^{2} \cap \mathbb{V}^{T} \varsubsetneqq \mathcal{G} .
$$

Proof. According to $(7)$ and the definition of $\mathbb{Q}^{2}$,

$$
\mathcal{G}=\bigcup_{Q \in \mathbb{N}} \bigcup_{a \in \mathbb{Z},(a, 2 Q)=1} \bigcup_{l \in \mathbb{Z}}\left\{\mathbf{y}=\left(\frac{a}{2 Q}, \frac{2 l+1-Q}{2 Q}\right)\right\} .
$$

First assume that $y \in \mathcal{G}$. To prove that $\mathbf{y} \in \mathbb{Q}^{2} \cap \mathbb{V}$, we need to find odd numbers $N$ and $M$ such that $\frac{2 l+1-Q}{2 Q}=N \frac{a}{2 Q}+\frac{M}{2}$, or $a N=2 l+1-(M+1) Q$. Since $(a, 2 Q)=1$, for every $l \in \mathbb{Z}$ there exists a unique $(\bmod 2 Q)$ solution $N=N_{l}$ of the congruence $a N \equiv 2 l+1$ $(\bmod 2 Q)$, which is the same, that there is a unique couple of integers $N=N_{l}$ and $s=s_{l}$ such that $N_{l} \in[0,2 Q)$ and $a N_{l}=2 l+1-2 s_{l} Q$. Since $a$ is odd, the numbers $N_{l}$ and $M_{l}:=2 s_{l}-1$ are also odd, so that $\mathbf{y} \in \mathbb{Q}^{2} \cap \mathbb{L}_{N_{l}, \frac{M_{l}}{2}} \in \mathbb{Q}^{2} \cap \mathbb{V}$. Therefore, $\mathcal{G} \subset \mathbb{Q}^{2} \cap \mathbb{V}$.

To prove the inverse inclusion, assume that $\mathbf{y}=\left(\frac{a}{q}, \frac{m}{q}\right) \in \mathbb{Q}^{2} \cap \mathbb{V}$. Then $(a, q)=1$, and there are such odd numbers $N, M$, that $\frac{m}{q}=N \frac{a}{q}+\frac{M}{2}$, or $2 m=2 N a+M q$. Since $M$ is odd, it follows from here that $q$ is an even number, $q=2 Q, Q \in \mathbb{N}$, and consequently, $m=a N+Q M$. Since both $a N$ and $M$ are odd numbers, it follows that $m+Q \equiv 1$ $(\bmod 2)$, so that according to $(7) G(\mathbf{y})=0$, or $\mathbf{y} \in \mathcal{G}$. This completes the proof of the equality a).

To prove the inclusion b), assume that $\mathbf{y}=\left(\frac{a}{q}, \frac{m}{q}\right) \in \mathbb{Q}^{2} \cap \mathbb{V}^{T}$. Then $(a, q)=1$, and there are such odd numbers $N, M$, that $\frac{a}{q}=N \frac{m}{q}+\frac{M}{2}$, or $2 a=2 N m+M q$. Consequently, $q$ is even, $q=2 Q, Q \in \mathbb{N}$, and $a=N m+M Q$. Since all three integers $a, N, M$ are odd, it follows that $m+Q \equiv 1(\bmod 2)$, so that according to $(7), \mathbf{y} \in \mathcal{G}$, which proves the inclusion $\mathbb{Q}^{2} \cap \mathbb{V}^{T} \subset \mathcal{G}$. Finally, it is easy to see that, in contrast to the equality a), the latter inclusion is strict, i.e. $\mathbb{Q}^{2} \cap \mathbb{V}^{T} \neq \mathcal{G}$. Indeed, consider the subset of $\mathbb{Q}^{2}$ consistent of the rational points of the form $\mathbf{y}=\left(\frac{a}{2 Q}, \frac{0}{2 Q}\right)$ where $Q$ is an odd natural number $\geq 3$, $a$ an odd integer, and $(a, Q)=1$. According to $(7)$, we have $G(\mathbf{y})=0$ for each such point, so that $\mathbf{y} \in \mathcal{G}$. The assumption $\left(\frac{a}{2 Q}, \frac{0}{2 Q}\right) \in \mathbb{V}^{T}$ would imply that there exist odd numbers $N, M$ such that $\frac{a}{2 Q}=N \frac{0}{2 Q}+\frac{M}{2}$, or $a=M Q$, which is in contradiction with the condition $(a, Q)=1$. This completes the proof of the lemma.

Proof of theorem 1. The density $\rho\left(f, \mathbb{R}^{2}\right)$ is a bivariate periodic function, and its trace $\rho(f, \mathbb{L})$ on a line $\mathbb{L}$ with a rational slope is a univariate function, also periodic. The weak limits in the claims of the theorem are constant functions. Therefore, we need to calculate the value of the limit, as $\varepsilon \rightarrow 0$, of the constant term of the Fourier expansions of $\rho\left(f, \mathbb{R}^{2}\right), \rho(f, \mathbb{L})$ for $f=f_{\varepsilon}$, and prove that each non-constant term of the expansion tends to 0 .

These limits will not depend on the selection of a concrete $\sqrt{\delta}$-family, or respectively $\sqrt{\delta_{+}}$-family of the initial data functions $\left\{f_{\varepsilon}\right\}_{\varepsilon>0}$. In the calculations below we apply our conventional notations $\sqrt{\delta}, \sqrt{\delta_{+}}$to emphasize in which sense the limits are understood. 
Thus, the relations

$$
\|\sqrt{\delta}\|_{\mathcal{L}^{2}\left(\mathbb{T}^{1}\right)}^{2}=1 ; \quad\|\sqrt{\delta}\|_{\mathcal{L}^{1}\left(\mathbb{T}^{1}\right)}=0 ; \quad \widehat{(\sqrt{\delta})}{ }_{n}=0
$$

mean that for every $\sqrt{\delta}$-family $\left\{f_{\varepsilon}\right\}$, and every fixed $n \in \mathbb{Z}$ we have, respectively,

$$
\int_{0}^{1}\left|f_{\varepsilon}(x)\right|^{2} d x \rightarrow 1, \quad \int_{0}^{1}\left|f_{\varepsilon}(x)\right| d x \rightarrow 0, \quad \int_{0}^{1} f_{\varepsilon}(x) e^{-2 \pi i n x} d x \rightarrow 0, \quad \varepsilon \rightarrow 0
$$

these relations are easy corollaries of the definition of $\sqrt{\delta}$-families. We also make a natural convention: for all non-integral $\alpha, \hat{f}_{\alpha}:=0$.

First of all, we have

$$
\rho(f ; t, x)=\sum_{(n, m) \in \mathbb{Z} \times \mathbb{Z}} \hat{f}_{n} \hat{f}_{m}^{*} e^{2 \pi i\left(\left(n^{2}-m^{2}\right) t+(n-m) x\right)}=\sum_{(n, m) \in \mathbb{Z} \times \mathbb{Z}} \hat{f}_{\frac{n+m}{2}} \hat{f}_{\frac{n-m}{2}}^{*} e^{2 \pi i(m(n t+x))},
$$

so that the Fourier coefficients $\hat{\rho}_{n, m}\left(f, \mathbb{T}^{2}\right),(n, m) \in \mathbb{Z}^{2}$, of $\rho\left(f, \mathbb{R}^{2}\right)$ are given by

$$
\begin{aligned}
& \hat{\rho}_{0,0}\left(f, \mathbb{T}^{2}\right)=\|f\|_{\mathcal{L}^{2}\left(\mathbb{T}^{1}\right)}^{2}-\left|\hat{f}_{0}\right|^{2} ; \quad \hat{\rho}_{n, 0}\left(f, \mathbb{T}^{2}\right)=0, n \in \mathbb{Z} \backslash\{0\} ; \\
& \hat{\rho}_{n, m}\left(f, \mathbb{T}^{2}\right)=\hat{f}_{\frac{1}{2}\left(\frac{n}{m}+m\right)} \hat{f}_{\frac{1}{2}\left(\frac{n}{m}-m\right)}^{*},(n, m) \in \mathbb{Z} \times(\mathbb{Z} \backslash\{0\}) .
\end{aligned}
$$

The claim $\rho\left(\sqrt{\delta}, \mathbb{R}^{2}\right)=1$ is a corollary from here and $(15)$, because $\hat{\rho}_{0,0}\left(\sqrt{\delta}, \mathbb{T}^{2}\right)=1$ and $\hat{\rho}_{n, m}\left(\sqrt{\delta}, \mathbb{T}^{2}\right)=0,(n, m) \neq(0,0)$.

Let us consider a line $\mathbb{L}_{N, \xi}=\left\{(t, x): x+N t=\xi, t \in \mathbb{R}^{1}\right\}$ with a rational slope $N=\frac{A}{Q}, Q \in \mathbb{N}^{1},(A, Q)=1$. Then the trace $\rho\left(f ; \mathbb{L}_{N, \xi}\right)$ is a periodic function, and its Fourier expansion is

$$
\begin{aligned}
& \rho\left(f ; \mathbb{L}_{N, \xi}\right)=\rho(f ; t,-N t+\xi) \sim \sum_{(\nu, \mu) \in \mathbb{Z} \times \mathbb{Z}} \hat{f}_{\frac{\nu+\mu}{2}} \hat{f}_{\frac{\nu-\mu}{2}}^{*} e^{2 \pi i(\mu(\nu t-N t+\xi))} \\
& =\sum_{n \in \mathbb{Z}} \hat{\rho}_{n}\left(f ; \mathbb{L}_{N, \xi}\right) e^{\frac{2 \pi i n t}{Q}}, \quad \hat{\rho}_{n}\left(f ; \mathbb{L}_{N, \xi}\right)=\sum_{\mu(\nu Q-A)=n} \hat{f}_{\frac{\nu+\mu}{2}} \hat{f}_{\frac{\nu-\mu}{2}}^{*} e^{2 \pi i \mu \xi} .
\end{aligned}
$$

If $n \neq 0$, then the set of solutions $\mu, \nu$ of the equation $\mu(\nu Q-A)=n$ is finite: both $\mu$ and $\nu Q-A$ have to be divisors of $n$. From here and (15) it follows that $\hat{\rho}_{n}\left(\sqrt{\delta}, \mathbb{L}_{N, \xi}\right)=0$ for $n \neq 0$.

Further, if $N \notin \mathbb{Z}$, then the solutions set $\{(\mu, \nu)\}$ of the equation $\mu(\nu Q-A)=0$ is $\{0\} \times \mathbb{Z}$, so that in this case

$$
\hat{\rho}_{0}\left(f ; \mathbb{L}_{N, \xi}\right)=\|f\|_{\mathcal{L}^{2}\left(\mathbb{T}^{1}\right)}^{2}, \quad \hat{\rho}_{0}\left(\sqrt{\delta}, \mathbb{L}_{N, \xi}\right)=1,
$$

whence we conclude that $\rho\left(\sqrt{\delta}, \mathbb{L}_{N, \xi}\right)=1$.

For an integer slope $N$, the solutions set $\{(\mu, \nu)\}$ of $\mu(\nu-N)=0$ is $(\{0\} \times \mathbb{Z}) \bigcup(\mathbb{Z} \times$ $\{N\}$ ), and consequently

$$
\begin{aligned}
& \hat{\rho}_{0}\left(f ; \mathbb{L}_{N, \xi}\right)=\sum_{\nu \in \mathbb{Z}}\left|\hat{f}_{\frac{\nu}{2}}\right|^{2}+\sum_{\mu \in \mathbb{Z}} \hat{f}_{\frac{N+\mu}{2}} \hat{f}_{\frac{N-\mu}{2}}^{*} e^{2 \pi i \mu \xi}-\left|\hat{f}_{\frac{N}{2}}\right|^{2} \\
& =\|f\|_{\mathcal{L}^{2}\left(\mathbb{T}^{1}\right)}^{2}+U_{N}(f, \xi)-\left|\hat{f}_{\frac{N}{2}}\right|^{2}, \quad U_{n}(f, x):=\sum_{m \in \mathbb{Z}} \hat{f}_{\frac{n+m}{2}} \hat{f}_{\frac{n-m}{2}}^{*} e^{2 \pi i m x} .
\end{aligned}
$$

$U_{n}(f, x)$ is the first of the following two analogs of Wigner's functions (see e.g. [17], 
Section 8.4 .3 (p. 357)), answering the periodic initial data:

$$
\begin{aligned}
& U_{n}(f ; x):=\int_{0}^{1} f(x+y) f^{*}(x-y) e^{-2 \pi i n y} d y, \\
& \tilde{U}_{n}(f ; x):=\int_{0}^{1} f(x+y) f^{*}(y-x) e^{-2 \pi i n y} d y, \quad n \in \mathbb{Z}, x \in \mathbb{R}^{1} .
\end{aligned}
$$

A direct calculation of the Fourier coefficients of these functions shows that

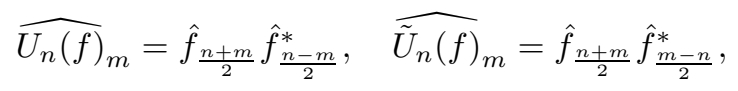

and consequently

$$
\sum_{n}\left\|U_{n}(f)\right\|_{\mathcal{L}^{2}\left(\mathbb{T}^{1}\right)}^{2}=\sum_{n}\left\|\tilde{U}_{n}(f)\right\|_{\mathcal{L}^{2}\left(\mathbb{T}^{1}\right)}^{2}=\|f\|_{\mathcal{L}^{2}\left(\mathbb{T}^{1}\right)}^{4}
$$

Wigner's functions are continuous, $\left\|U_{n}(f)\right\|_{\mathbb{C}\left(\mathbb{T}^{1}\right)},\left\|\tilde{U}_{n}(f)\right\|_{\mathbb{C}\left(\mathbb{T}^{1}\right)} \leq\|f\|_{\mathcal{L}^{2}\left(\mathbb{T}^{1}\right)}^{2}$, and $U_{n}$ is real-valued for every $f \in \mathcal{L}^{2}\left(\mathbb{T}^{1}\right)$. The latter is not true for $\tilde{U}_{n}$ in a general case, but if $f$ is even, then obviously $U_{n}(f)=\tilde{U}_{n}(f)$; if, on the contrary, $f$ is odd, then $U_{n}(f)=-\tilde{U}_{n}(f)$.

It is well known that Wigner's functions are intrinsically related with the density $\rho$. They appear, see (16), as profiles of the plane waves in ridge series representation of $\rho$ on $\mathbb{R}^{2}$

$$
\rho(f)=\sum_{n} \mathcal{U}_{n}(f), \quad \mathcal{U}_{n}(f):=U_{n}(f ; n t+x),
$$

and simultaneously as Fourier coefficients of the expansion of $\rho$ on the lines, parallel to $x$-axis:

$$
\rho(f ; t, x)=\sum_{m \in \mathbb{Z}} \tilde{U}_{m}(f ; m t) e^{2 \pi i m x} .
$$

Since

$$
\int_{0}^{1} \int_{0}^{1} \mathcal{U}_{n}(f) \mathcal{U}_{m}(f) d t d x= \begin{cases}\left|\hat{f}_{\frac{n}{2}} \hat{f}_{\frac{m}{2}}\right|^{2}, & \text { if } n \neq m \\ \left\|U_{n}(f)\right\|_{\mathcal{L}^{2}\left(\mathbb{T}^{1}\right)}, & \text { if } n=m\end{cases}
$$

it follows that the ridge series in (20) converges unconditionally in the space $\mathcal{L}^{2}\left(\mathbb{T}^{2}\right)$. Indeed, the double integral on the left side equals the inner product $\left(\mathcal{U}_{n}, \mathcal{U}_{m}\right)_{\mathcal{L}^{2}\left(\mathbb{T}^{2}\right)}$ of $\mathcal{U}_{n}$ and $\mathcal{U}_{m}$ in $\mathcal{L}^{2}\left(\mathbb{T}^{2}\right)$. Therefore, for an arbitrary subset of integers $\mathbb{A} \subset \mathbb{Z}$ we have

$$
\left\|\sum_{n \in \mathbb{A}} \mathcal{U}_{n}\right\|_{\mathcal{L}^{2}\left(\mathbb{T}^{2}\right)}^{2}=\sum_{(n, m) \in \mathbb{A} \times \mathbb{A}}\left(\mathcal{U}_{n}, \mathcal{U}_{m}\right)_{\mathcal{L}^{2}\left(\mathbb{T}^{2}\right)} \leq 2\|f\|_{\mathcal{L}^{2}\left(\mathbb{T}^{1}\right)}^{4}-\sum_{n \in \mathbb{Z}}\left|\hat{f}_{n}\right|^{4} .
$$

This implies the unconditional convergence, and along with it, the following variant of the Strichartz' inequality ${ }^{5}$ for the $\mathcal{L}^{4}$-norm of the solution $\psi$ on $\mathbb{T}^{2}$ :

$$
\|\rho(f)\|_{\mathcal{L}^{2}\left(\mathbb{T}^{2}\right)}^{1 / 2}=\|\psi(f)\|_{\mathcal{L}^{4}\left(\mathbb{T}^{2}\right)} \leq 2^{1 / 4}\|f\|_{\mathcal{L}^{2}\left(\mathbb{T}^{1}\right)} .
$$

\footnotetext{
${ }^{4}$ In the recent literature on approximation theory, see e.g. [30], the term ridge pinpoints the function of the type plane wave, as a tool of approximation of a general multi-variate function. In particular, ridge series simply means an infinite sum of plane waves.

${ }^{5}$ The estimates of the integral norms of the oscillatory solutions, as functions of both space and time variables, via the integral norms of the initial data are often called in the modern literature Strichartz' type inequalities.
} 
According to (18), the average values (Radon transformations) of $\rho$ on the lines $\mathbb{L}_{N, \xi}$ with the integral slopes $N$ are expressed via Wigner's functions, too. Their limits for $\sqrt{\delta}, \sqrt{\delta_{+}}$-families are the following:

$$
U_{n}\left(\sqrt{\delta_{+}}, x\right)=-U_{n}\left(\sqrt{\delta_{-}}\right)=\tilde{U}_{n}(\sqrt{\delta}, x)= \begin{cases}0, & \text { if } 2 x \notin \mathbb{Z}, \\ (-1)^{2 n x}, & \text { if } 2 x \in \mathbb{Z} .\end{cases}
$$

Indeed,

$$
\tilde{U}_{n}(f, x)=e^{2 \pi i n x} \int_{0}^{1} f(y) f^{*}(y-2 x) e^{-2 \pi i n y} d y .
$$

Consider a $\sqrt{\delta}$-family $\mathcal{F}=\left\{f_{\varepsilon}\right\}_{\varepsilon}>0$; then we see that if $2 x \notin \mathbb{Z}$, then $\tilde{U}_{n}\left(f_{\varepsilon}, x\right) \rightarrow 0, \varepsilon \rightarrow$ 0 ; on the contrary, if $2 x \in \mathbb{Z}$, then $\tilde{U}_{n}\left(f_{\varepsilon}, x\right) \rightarrow e^{2 \pi i n x}=(-1)^{2 n x}$. If $\mathcal{F}$ is $\sqrt{\delta_{+}}$-family, then, as mentioned above, $U_{n}\left(f_{\varepsilon}\right)=\tilde{U}_{n}\left(f_{\varepsilon}\right)$; for a $\sqrt{\delta_{-}}$-family, $U_{n}\left(f_{\varepsilon}\right)=-\tilde{U}_{n}\left(f_{\varepsilon}\right)$, and (22) follows.

From this and from (18) we conclude that for a line $\mathbb{L}_{N, \xi}$ with the integral slope $N$

$$
\rho\left(\sqrt{\delta_{ \pm}}, \mathbb{L}_{N, \xi}\right)=\hat{\rho}_{0}\left(\sqrt{\delta_{ \pm}}, \mathbb{L}_{N, \xi}\right)=1 \pm \tilde{U}_{N}(\sqrt{\delta}, \xi)= \begin{cases}1, & \text { if } 2 \xi \notin \mathbb{Z}, \\ 1 \pm(-1)^{2 N \xi}, & \text { if } 2 \xi \in \mathbb{Z},\end{cases}
$$

and to finish the proof of theorem 1, it remains to consider the weak limits on the lines $\mathbb{L}^{T}(0, \tau)=\{(t, x): t=\tau\}$, parallel to the $x$-axis. If $\tau$ is an irrational number, then according to $(21),(22)$ we have $\tilde{U}_{m}(\sqrt{\delta}, m \tau)=0, m \neq 0 ; \tilde{U}_{0}(\sqrt{\delta}, 0)=1$, and the equality $\rho\left(\sqrt{\delta}, \mathbb{L}^{T}(0, \tau)\right)=1$ follows from (21). Finally, if $\tau$ is a rational number, then (4) is a corollary of (6), and the classical formulas (7) for the magnitudes of the Gauss sums. But we can also derive (4), using (22) and (21):

$$
\rho\left(\sqrt{\delta}, \mathbb{L}^{T}(0, \tau)\right)=\sum_{m} \tilde{U}_{m}(\sqrt{\delta}, m \tau) e^{2 \pi i m x}=\sum_{2 m \tau \in \mathbb{Z}}(-1)^{2 m^{2} \tau} e^{2 \pi i m x} .
$$

Assume that $\tau=\frac{a}{q}, a \in \mathbb{Z}, q \in \mathbb{N}^{1},(a, q)=1$. If $q$ is odd, then $2 m \tau \in \mathbb{Z}$ means that $m \equiv 0(\bmod q)$, and in this case $(-1)^{2 m^{2} \tau}=1$, so that

$$
\rho\left(\sqrt{\delta}, \mathbb{L}^{T}(0, \tau)\right)=\sum_{m} e^{2 \pi i m q x}=\frac{1}{q} \sum_{n} \delta_{\frac{n}{q}} .
$$

If $q$ is even, $q=2 Q, Q \in \mathbb{N}^{1}$, then $2 m \tau \in \mathbb{Z}$ means that $m \equiv 0(\bmod Q)$. In this case $(-1)^{2 m^{2} \tau}=(-1)^{m^{2} Q}=(-1)^{m Q}=e^{\pi i m Q}$, and thus

$$
\rho\left(\sqrt{\delta}, \mathbb{L}^{T}(0, \tau)\right)=\sum_{m} e^{2 \pi i m Q(x+1 / 2)}=\frac{1}{Q} \sum_{n} \delta_{\frac{1}{2}+\frac{n}{Q}}=\frac{1}{q} \sum_{n}\left(1+(-1)^{n+\frac{q}{2}}\right) \delta_{\frac{n}{q}} .
$$

This completes the proof of theorem 1 , and remark 1 .

7. The exponential sums with the quadratic phase. The known results concerning the sums $W_{n}(\mathbf{x})$, see section 3 , in the generic case $r=2$ are much more detailed, than for $r \geq 3$. A fundamental input in the analysis of the exponential sums with the quadratic polynomial phase was done by G. H. Hardy and J. E. Littlewood in [15]. With the global precision (error estimate) of order $O\left(n^{-1 / 2}\right), n \rightarrow \infty$, the asymptotic formulas of Vinogradov's type (10) reach out everywhere on $\mathbb{R}^{2}$. In this sense, the minor arcs for $r=2$ have been "eliminated", see [13], or [26]. 
Given a natural number $\nu$, let us denote $\mathbb{Q}_{\nu}^{2}:=\left\{\mathbf{y} \in \mathbb{Q}^{2}: q(\mathbf{y}) \leq \nu\right\}$, i .e., the subset of rational points $\mathbf{y}=\left(\frac{a}{q}, \frac{m}{q}\right) \in \mathbb{Q}^{2}$ (recall, that this notation implies that $(a, q)=1$ ), such that $q \leq \nu$; further, let

$$
\square_{\nu}^{2}(\mathbf{y}):=\left\{\mathbf{y}+\mathbf{z}, \quad\left|z_{2}\right| \leq \frac{1}{\nu q}, \quad\left|z_{1}\right| \leq \frac{1}{2 q}\right\}, \quad q=q(\mathbf{y}), \mathbf{y} \in \mathbb{Q}_{\nu}^{2} .
$$

Then

(i) $W_{n}(\mathbf{y}+\mathbf{z})-G(\mathbf{y}) \mathcal{W}_{n}(\mathbf{z})=O\left(\frac{\sqrt{q}}{n}\right)=O\left(\frac{1}{\sqrt{n}}\right), \mathbf{y}+\mathbf{z} \in \square_{8 n}^{2}(\mathbf{y})$;

(ii) $\bigcup_{\mathbf{y} \in \mathbb{Q}_{8 n}^{2}} \square_{8 n}^{2}(\mathbf{y})=\mathbb{R}^{2}$.

The proof of $(23, \mathrm{i})$ in [13], [26] followed a general scheme that originated from the papers of Vinogradov and Van der Corput. Further, (23,ii) says that every point $\mathbf{x}$ on $\mathbb{R}^{2}$ belongs to a rectangle $\square_{8 n}^{2}(\mathbf{y})$ with $q=q(\mathbf{y}) \leq 8 n$, where the asymptotic formula (23,i) is valid for $W_{n}(\mathbf{x})$. (23,ii) it is a corollary from the Dirichlet box principle (also known as pigeon hole). Indeed, let $\mathbf{x}=(t, x) \in \mathbb{R}^{2}, \nu \in \mathbb{N}$. Then there exists a rational number $y_{2}=\frac{a}{q},(a, q)=1$ such that $\left|z_{2}\right| \leq \frac{1}{\nu q}, z_{2}:=t-y_{2}$, and $q \leq \nu$. Once such $y_{2}$ is established, we can find an integer $m$ (in general, not co-prime with $q$ ) such that for $y_{1}:=\frac{m}{q}, z_{1}:=x-y_{1}$ we have $\left|z_{1}\right| \leq \frac{1}{2 q}$. Summarizing, we see that every $\mathbf{x} \in \mathbb{R}^{2}$ belongs to a rectangle $\square_{n}^{2}(\mathbf{y})$ where $q=q(\mathbf{y}) \leq \nu:=8 n$, which implies $(23$, ii $)$.

The rational approximant $\frac{a}{q}$ of $t$ can be also found more "constructively", as a convergent fraction $[t]+\frac{a_{j}}{q_{j}}$, where $[t]$ denotes the integer part of $t$, and $\frac{a_{j}}{q_{j}}-$ the $j$ th truncation, $\frac{a_{j}}{q_{j}}:=\left[k_{1}, \ldots, k_{j}\right], j=1,2, \ldots$, of the full continued fraction of $\{t\}=t-[t]$, see e.g. [18], Ch. 10,

$$
\{t\}=\frac{1}{k_{1}+\frac{1}{k_{2}+\cdots}}=\left[k_{1}, k_{2}, \ldots\right], \quad k_{j} \in \mathbb{N}, j=1,2, \ldots
$$

The natural numbers $k_{j}$ are known as the partial quotients of $t$, and the sequence of the convergents $\left\{\frac{a_{j}}{q_{j}}\right\}$ is defined by the matrix relations

$$
\left(\begin{array}{cc}
a_{j+1} & a_{j} \\
q_{j+1} & q_{j}
\end{array}\right)=\left(\begin{array}{cc}
a_{j} & a_{j-1} \\
q_{j} & q_{j-1}
\end{array}\right)\left(\begin{array}{cc}
k_{j} & 1 \\
1 & 0
\end{array}\right), j=1, \ldots ; \quad\left(\begin{array}{ll}
a_{1} & a_{0} \\
q_{1} & q_{0}
\end{array}\right):=\left(\begin{array}{cc}
1 & 0 \\
k_{1} & 1
\end{array}\right) .
$$

All fractions $\frac{a_{j}}{q_{j}}$ generated by this recurrence are reduced, i.e. $\left(a_{j}, q_{j}\right)=1$; the approximation properties with regard to the given $t \in \mathbb{R}^{1}$ are the following:

$$
t=[t]+\frac{a_{j}}{q_{j}}+\frac{(-1)^{j} \theta_{j}}{q_{j} q_{j+1}}, \quad \frac{1}{2} \leq \theta_{j} \leq 1 ; \quad j=0,1, \ldots
$$

The following lemma is true.

Lemma 2. Assume that $N, M$ are odd integers, $n$ a natural number, $\varepsilon>0$. Denote by $\tilde{\mathbb{L}}_{N, M, \varepsilon}$, and respectively $\tilde{\mathbb{L}}_{N, M, \varepsilon}^{T}$, the following stripes around the lines $\mathbb{L}_{N, M}, \mathbb{L}_{N, M}^{T}$ : 


$$
\begin{aligned}
& \tilde{\mathbb{L}}_{N, M, \varepsilon}:=\left\{\mathbf{x}=(t, x) \in \mathbb{R}^{2}, \quad x=N t+\frac{M}{2}+\delta,|\delta| \leq \varepsilon\right\}, \\
& \tilde{\mathbb{L}}_{N, M, \varepsilon}^{T}:=\left\{\mathbf{x}=(t, x) \in \mathbb{R}^{2}, \quad x=\frac{1}{N}\left(t-\frac{M}{2}\right)+\delta,|\delta| \leq \frac{\varepsilon}{|N|}\right\} .
\end{aligned}
$$

Then for $n \geq|N|, \varepsilon=\varepsilon_{n}:=\frac{1}{32 n}$

$$
\text { (i) } \sup _{(t, x) \in \tilde{\mathbb{L}}_{N, M, \varepsilon}}\left|\sum_{\nu=1}^{n} e^{2 \pi i\left(\nu^{2} t+\nu x\right)}\right| \leq c \sqrt{n}, \quad \text { (ii) } \sup _{(t, x) \in \tilde{\mathbb{L}}_{N, M, \varepsilon}^{T}}\left|\sum_{\nu=1}^{n} e^{2 \pi i\left(\nu^{2} t+\nu x\right)}\right| \leq c \sqrt{|N| n} \text {. }
$$

Proof. We will derive this lemma from (23). Recall that

$$
W_{n}(\mathbf{x})=\frac{1}{n} \sum_{\nu=1}^{n} e^{2 \pi i\left(\nu^{2} t+\nu x\right)}
$$

Let us first prove $(26, \mathrm{i})$, i.e. that if $n \geq|N|, \mathbf{x}=(t, x) \in \tilde{\mathbb{L}}_{N, M, \varepsilon}$, where $N, M$ are odd, then $\left|W_{n}(\mathbf{x})\right| \leq \frac{c}{\sqrt{n}}$. Without loss of generality, we may assume that $t$ is an irrational number on $(0,1)$.

Further, by (23), the main terms $G(\mathbf{y}) \mathcal{W}_{n}(\mathbf{z})$ only have to be estimated. In the other words, we have to show that for a sufficiently large absolute constant $c$,

$$
\tilde{\mathbb{L}}_{N, M, \varepsilon} \subset \bigcup_{\mathbf{y} \in \mathbb{Q}_{8 n}^{2}}\left\{\mathbf{y}+\mathbf{z} \in \square_{8 n}^{2}(\mathbf{y}),\left|G(\mathbf{y}) \mathcal{W}_{n}(\mathbf{z})\right| \leq \frac{c}{\sqrt{n}}\right\}
$$

Given $\mathbf{x}=(t, x) \in \tilde{\mathbb{L}}_{N, M, \varepsilon}$ with an irrational $t \in(0,1)$, consider the sequence of convergent fractions $\left\{\frac{a_{j}}{q_{j}}\right\}$ of $t$, see $(24)$, and find $j$ such that $8 n \in\left[q_{j}, q_{j+1}\right)$. Denote

$$
q:=q_{j}, \bar{q}:=q_{j+1}, \quad y_{2}:=\frac{a}{q}=\frac{a_{j}}{q_{j}}, y_{1}:=\frac{\bar{m}}{q} ; \quad \mathbf{y}:=\left(y_{2}, y_{1}\right) ; \quad \mathbf{z}:=\mathbf{x}-\mathbf{y},
$$

where the integer $\bar{m}$ is determined by the condition $-\frac{1}{2 q} \leq x-\frac{\bar{m}}{q}<\frac{1}{2 q}$. Then $\mathbf{x} \in \square_{8 n}^{2}(\mathbf{y})$, where $q(\mathbf{y})=q=q_{j} \leq 8 n$, because $\left|z_{1}\right| \leq \frac{1}{2 q}$ by the definition of $\bar{m}$, and $\left|z_{2}\right| \leq \frac{1}{q \bar{q}}<\frac{1}{8 n q}$.

To prove that $\left|G(\mathbf{y}) \mathcal{W}_{n}(\mathbf{z})\right| \leq c n^{-1 / 2}$, let us recall that for $\mathbf{x}=(t, x) \in \tilde{\mathbb{L}}_{N, M, \varepsilon}$ we have

$$
x=N t+\frac{M}{2}+\delta,|\delta| \leq \frac{1}{32 n},
$$

and consider separately two cases: a) $q=q(\mathbf{y})$ is even, and b) $q$ is odd.

In the case a) we have $q=2 Q, Q \in \mathbb{N}^{1}$, and

$$
\left|x-\frac{N a+M Q}{q}\right| \leq \frac{|N|}{q \bar{q}}+|\delta| \leq \frac{|N|}{8 n q}+\frac{1}{32 n} \leq \frac{1}{8 q}+\frac{1}{4 q}<\frac{1}{2 q},
$$

whence it follows that $\bar{m}=N a+M Q$. Consequently, in the case a) the point $\mathbf{y}$, defined by (28), belongs to the line $\mathbb{L}_{N, M}$. On this line, according to lemma 1, all Gauss sums $G(\mathbf{y})$ vanish, so that $G(\mathbf{y}) \mathcal{W}_{n}(\mathbf{z})=0$, which completes the consideration of the case a).

In the case b) the denominator $q$ is an odd number, and therefore, the magnitude of $z_{1}$ is estimated from below as $\left|z_{1}\right| \geq \frac{1}{8 q}$, because 


$$
\begin{aligned}
\left|z_{1}\right|= & \min _{m \in \mathbb{Z}}\left|x-\frac{m}{q}\right|=\min _{m \in \mathbb{Z}}\left|N t+\frac{M}{2}+\delta-\frac{m}{q}\right| \\
& \geq \min _{m \in \mathbb{Z}}\left|\frac{1}{2}-\frac{m}{q}\right|-\left(\frac{|N|}{q \bar{q}}+|\delta|\right) \geq \frac{1}{2 q}-\left(\frac{1}{8 q}+\frac{1}{4 q}\right) \geq \frac{1}{8 q} .
\end{aligned}
$$

Therefore, by (11), the oscillatory integral $\mathcal{W}_{n}(\mathbf{z})$ satisfies the estimate

$$
\left|\mathcal{W}_{n}(\mathbf{z})\right| \leq \frac{c}{\sqrt{\left|z_{2}\right| n^{2}+\left|z_{1}\right| n}} \leq \frac{c \sqrt{q}}{\sqrt{n}}
$$

and we have $|G(\mathbf{y})|=\frac{1}{\sqrt{q}}$. Thus, $\left|G(\mathbf{y}) \mathcal{W}_{n}(\mathbf{z})\right| \leq \frac{c}{\sqrt{n}}$, which completes the proof of (27), and $(26, \mathrm{i})$.

To prove the second part of the lemma, i.e., the estimate $(26, \mathrm{ii})$, it is sufficient to establish the following modification of (27):

$$
\tilde{\mathbb{L}}_{N, M, \varepsilon}^{T} \subset \bigcup_{\mathbf{y} \in \mathbb{Q}_{8 n}^{2}}\left\{\mathbf{y}+\mathbf{z} \in \square_{8 n}^{2}(\mathbf{y}),\left|G(\mathbf{y}) \mathcal{W}_{n}(\mathbf{z})\right| \leq \frac{c \sqrt{|N|}}{\sqrt{n}}\right\} .
$$

For $\mathbf{x}=(t, x) \in \tilde{\mathbb{L}}_{N, M, \varepsilon}^{T}$ with an irrational $t$, let us modify the rational point $\mathbf{y}$ in (28) by letting $y_{2}=\frac{a}{q}:=[t]+\frac{a_{j}}{q_{j}}$, where $j$ is determined as above, i.e., by the condition $8 n \in\left[q_{j}, q_{j+1}\right)$, and $y_{1}:=\frac{\bar{m}}{q}$, also as above, by the condition $-\frac{1}{2 q} \leq x-\frac{\bar{m}}{q}<\frac{1}{2 q}$. Then $\mathbf{x} \in \square_{8 n}^{2}(\mathbf{y})$, and it follows from the definition of the stripe $\tilde{\mathbb{L}}_{N, M, \varepsilon}^{T}$ that

$$
t=\frac{a}{q}+\frac{\theta}{q \bar{q}},|\theta| \leq 1, \quad x=\frac{2 a-q M}{2 q N}+\frac{\theta}{N q \bar{q}}+\delta, \quad|\delta| \leq \frac{1}{32 n|N|} .
$$

Let

$$
\zeta:=\min _{m \in \mathbb{Z}}\left|\frac{2 a-q M}{2 q N}-\frac{m}{q}\right|,
$$

and denote $\tilde{m}$ the integer, for which this minimum is attained; let $\tilde{\mathbf{y}}:=\left(\frac{a}{q}, \frac{\tilde{m}}{q}\right)$.

There are two possibilities: either a) $\zeta=0$, or b) $\zeta \geq \frac{1}{2 q|N|}$.

Let us first consider the case a). In this case, $q$ is necessarily an even number, i.e. $q=2 Q$, and we have $a=Q M+N \tilde{m}$. All three numbers $a, M, N$ are odd, so that if $Q$ is even, then $\tilde{m}$ is odd; on the contrary, if $Q$ is odd, then $\tilde{m}$ is even. Anyway, in the case a) $Q-\tilde{m} \equiv 1(\bmod 2)$, and according to lemma $1 G(\tilde{\mathbf{y}})=0$. It is easy to see that $\tilde{m}=\bar{m}$, i.e. $\tilde{\mathbf{y}}=\mathbf{y}$, because

$$
\left|x-\frac{\tilde{m}}{q}\right| \leq \zeta+\frac{1}{|N| q \bar{q}}+\frac{1}{32 n|N|} \leq \frac{1}{4 q} .
$$

This means, that in the case a) the point $\mathbf{y}$ belongs to the transversal line $\mathbb{L}_{N, M}^{T}$. This completes consideration of the case a), because $G(\mathbf{y}) \mathcal{W}_{n}(\mathbf{z})=0$.

In the case b), the magnitude of $z_{1}$ is estimated from below as follows:

$$
\left|z_{1}\right| \geq \zeta-\left(\frac{1}{|N| q \bar{q}}+\frac{1}{32 n|N|}\right) \geq \frac{1}{|N|}\left(\frac{1}{2 q}-\frac{1}{4 q}\right) \geq \frac{1}{4|N| q} .
$$

Consequently, by (29), the oscillatory integral $\mathcal{W}_{n}$ in the case b) satisfies the estimate $\left|\mathcal{W}_{n}(\mathbf{z})\right| \leq c \sqrt{\frac{|N| q}{n}}$, and since $|G(\mathbf{y})| \leq \sqrt{\frac{2}{q}}$, we see that in this case $\left|G(\mathbf{y}) \mathcal{W}_{n}(\mathbf{z})\right| \leq$ $c \sqrt{\frac{|N|}{n}}$, which completes the proof of the lemma. 
For $\varepsilon:=\frac{1}{2 n}$ we have

$$
\psi\left(w_{\varepsilon} ; t, x\right)=\frac{1}{\sqrt{2 n}} \sum_{|\nu| \leq n} e^{2 \pi i\left(\nu^{2} t+\nu x\right)}=\sqrt{\frac{n}{2}}\left(W_{n}(t, x)+W_{n}(t,-x)-\frac{1}{n}\right) .
$$

Thus, the claim of theorem 2 concerning boundedness of the density $\rho\left(w_{\varepsilon} ; t, x\right)$ on the lines $\mathbb{L}_{N, M}, \mathbb{L}_{N, M}^{T}$ with odd $N, M$ is a corollary from lemma 4 .

Remark 3 to theorem 2 is also a corollary from the asymptotic formula (23). This statement is of the type of $\Omega$-theorems, established by Hardy and Littlewood in [15], and we do not provide the detailed proof here. The set

$$
\Xi=\left\{\xi=\left[k_{1}, k_{2}, \ldots\right] ; \limsup _{j \rightarrow \infty} k_{j}=\infty\right\},
$$

i.e., the collection of all $\xi$ with unbounded partial quotients, see (24), provides the exact description of the "bad" values of the parameter $\xi$.

8. Hilbert transforms. Global boundedness, self-similarity. The following results concerning discrete oscillatory Hilbert transforms with the algebraic polynomial phase were proved by Arkhipov and the author in [2] on the base of Vinogradov's method of exponential sums:

$$
\begin{aligned}
& \text { (i) } \sup _{n \in \mathbb{N}^{1}} \sup _{\mathbf{x} \in \mathbb{R}^{r}}\left|H_{n}(\mathbf{x})\right|<\infty, H_{n}(\mathbf{x}):=\sum_{1 \leq|\nu| \leq n} \frac{e^{2 \pi i P(\mathbf{x}, \nu)}}{2 \pi i \nu}, \\
& \text { (ii) } \forall \mathbf{x} \in \mathbb{R}^{r}, \quad \exists H(\mathbf{x}):=\lim _{n \rightarrow \infty} H_{n}(\mathbf{x})=\text { p.v. } \sum_{\nu \in \mathbb{Z} \backslash\{0\}} \frac{e^{2 \pi i P(\mathbf{x}, \nu)}}{2 \pi i \nu} .
\end{aligned}
$$

Independently and somewhat later the global boundedness result was proved by E. M. Stein and S. Wainger, see [34]. Let us note that in a much earlier paper [33] Stein and Wainger established boundedness of the integral analogs of $H$, i.e.

$$
\forall r \in \mathbb{N}: \quad\|\mathcal{H}\|_{\mathcal{L}^{\infty}\left(\mathbb{R}^{r}\right)}<\infty, \quad \mathcal{H}(\mathbf{x}):=\text { p.v. } \int_{\mathbb{R}^{1}} \frac{e^{2 \pi i P(\mathbf{x}, \nu)}}{2 \pi i \nu} d \nu .
$$

The function $H(\mathbf{x})$ is self-similar which can be expressed, see [28], by the following local incremental relations, see also (10):

$$
\text { (i) } H(\mathbf{z})=\mathcal{H}(\mathbf{z})+o(1) ; \quad \text { (ii) } H(\mathbf{y}+\mathbf{z})-H(\mathbf{y})=G(\mathbf{y}) \mathcal{H}(\mathbf{z})+o(1), \mathbf{z} \rightarrow \mathbf{0}, \quad \mathbf{y} \in \mathbb{Q}^{r},
$$

and for $\mathbf{y} \in \mathbb{Q}^{r}$ the value of $H(\mathbf{y})$ can be calculated as a finite discrete Hilbert transformation, which is an analog of the complete rational sum $G(\mathbf{y})$ :

$$
H(\mathbf{y})=\frac{1}{2 q i} \sum_{n=1}^{q-1} e^{2 \pi i P(\mathbf{y}, n)} \cot \frac{\pi n}{q} .
$$

Obviously, in (32) the complete rational sums again appear as the scaling factors, while the pattern is represented by the integral Hilbert transformation $\mathcal{H}$.

The author's original goal of consideration of the transforms $H_{N}$ was P. L. Ul'yanov's [36] problem concerning the so-called spectra of uniform convergence, see [28]. The initial result [23] was the following estimate ( "à la H. Weyl", cf. [38]), of the finite transforms 
$H_{N}$, uniform in the coefficients of the algebraic polynomial in the exponent:

$$
\left\|H_{n}\right\|_{\mathcal{L}^{\infty}\left(\mathbb{R}^{r}\right)}=O\left((\ln n)^{1-\varepsilon_{r}}\right), \quad n \rightarrow \infty, \quad \varepsilon_{r}=2^{1-r} .
$$

This estimate was sufficient to solve the problem raised by Ul'yanov in [36]. Namely, consider an algebraic polynomial $Q$ of degree $r \geq 1$ whose coefficients are natural numbers. Denote by $\mathcal{C}\left(Q, \mathbb{T}^{1}\right)$ the subspace of continuous periodic functions $\mathcal{C}\left(\mathbb{T}^{1}\right)$ whose Fourier coefficients vanish outside of the polynomial sequence $\mathcal{Q}:=\{Q(n)\}_{n \in \mathbb{Z}}$. Ul'yanov asked whether or not among polynomial sequences $\mathcal{Q}$ of degree $r \geq 2$ there exists a spectrum of uniform convergence. In other words, does there exist a polynomial $Q$ such that the Fourier series converges uniformly for all functions of the subspace $\mathcal{C}\left(Q, \mathbb{T}^{1}\right)$ ? It follows from the above estimate that the answer to this problem is in the negative, and the classical result of P. du Bois Reymond, see e.g. [40], Ch. 8, on the existence of a continuous function whose Fourier series diverges at a point, can be extended to all subspaces $\mathcal{C}\left(Q, \mathbb{T}^{1}\right)$. For more details, the reader is referred to [23], or to the review paper [28].

After the more refined result (31) was obtained, Z. Ciesielski drew the author's attention to the possible applications in the study of the properties of the solutions of Schrödinger type equations.

A corollary of (31) is the global boundedness condition for $V$-extensions. Denote by $\mathcal{B V}\left(\mathbb{T}^{1}\right)$ the space of univariate periodic functions, of period $=1$, whose total variation in the usual sense is bounded on $[0,1)$, with the norm $\|f\|_{\mathcal{B V}}:=\operatorname{var}(f,[0,1))+|f(0)|$. Then $V: \mathcal{B V}\left(\mathbb{T}^{1}\right) \rightarrow \mathcal{L}^{\infty}\left(\mathbb{R}^{r}\right)$ in the following sense:

$$
\begin{aligned}
& \sup _{n \in \mathbb{N}^{1}}\left\|V_{n}(f ; \cdot)\right\|_{\mathcal{L}^{\infty}\left(\mathbb{R}^{r}\right)} \leq c\|f\|_{\mathcal{B} \mathcal{V}}, \quad V_{n}(f ; \mathbf{x}):=\sum_{|\nu| \leq n} \hat{f}_{\nu} e^{2 \pi i P(\mathbf{x}, \nu)} ; \\
& \forall f \in \mathcal{B} \mathcal{V}\left(\mathbb{T}^{1}\right), \quad \forall \mathbf{x} \in \mathbb{R}^{r} \quad \exists \lim _{n \rightarrow \infty} V_{n}(f ; \mathbf{x}):=\text { p.v. } \sum_{\nu \in \mathbb{Z}} \hat{f}_{\nu} e^{2 \pi i P(\mathbf{x}, \nu)} .
\end{aligned}
$$

A further corollary is the existence of generalized solutions of the Cauchy initial data problem for every Schrödinger type equation with the constant coefficients in the class of regular and everywhere bounded functions. Thus, assume that $P$ is a univariate algebraic polynomial with the real coefficients, and consider the Cauchy initial value problem

$$
\frac{1}{2 \pi i} \frac{\partial \Psi}{\partial t}=P\left(\frac{1}{2 \pi i} \frac{\partial}{\partial x}\right) \Psi,\left.\quad \Psi(t, x)\right|_{t=0}=f(x) .
$$

If $f \in \mathcal{B V}\left(\mathbb{T}^{1}\right)$, then the generalized solution of this problem

$$
\Psi=\Psi(f ; P ; t, x)=\text { p.v. } \sum_{n \in \mathbb{Z}} \hat{f}_{n} e^{2 \pi i(t P(n)+n x)}
$$

is a regular and everywhere bounded function.

For $r=2$, the result $H \in \mathcal{L}^{\infty}\left(\mathbb{T}^{2}\right)$ is equivalent to the global boundedness of the solution

$$
\psi(t, x)=\mathrm{p} . \mathrm{v} . \sum_{n \in \mathbb{Z} \backslash\{0\}} \frac{e^{2 \pi i\left(n^{2} t+n x\right)}}{2 \pi i n}=H(t, x)
$$


of the problem

$$
\frac{\partial \psi}{\partial t}=\frac{1}{2 \pi i} \frac{\partial^{2} \psi}{\partial x^{2}},\left.\quad \psi(t, x)\right|_{t=0}=\frac{1}{2}-\{x\}=\text { p.v. } \sum_{n \in \mathbb{Z} \backslash\{0\}} \frac{e^{2 \pi i n x}}{2 \pi i n}=\sum_{n \in \mathbb{N}^{1}} \frac{\sin 2 \pi n x}{\pi n} .
$$

If the initial data function is the window function (Ronchi's grating), $f(x)=\tilde{\mathbf{1}}_{\varepsilon}(x)$, see (2), then

$$
\psi\left(\tilde{\mathbf{1}}_{\varepsilon}, t, x\right)=\frac{1}{\sqrt{\varepsilon}} \sum_{n \in \mathbb{Z}} \frac{\sin \pi n \varepsilon}{\pi n} e^{2 \pi i\left(n^{2} t+n x\right)}=\sqrt{\varepsilon}+\frac{H(t, x+\varepsilon / 2)-H(t, x-\varepsilon / 2)}{\sqrt{\varepsilon}} .
$$

Therefore, the first conclusion from $H \in \mathcal{L}^{\infty}\left(\mathbb{T}^{2}\right)$ is that for each fixed $\varepsilon>0$ the solution $\psi\left(\tilde{\mathbf{1}}_{\varepsilon}\right)$ is an everywhere bounded function on $\mathbb{R}^{2}$, and for the corresponding density we have $\left\|\rho\left(\tilde{\mathbf{1}}_{\varepsilon}\right)\right\|_{\mathcal{L}^{\infty}\left(\mathbb{R}^{2}\right)}=O\left(\varepsilon^{-1}\right), \varepsilon \rightarrow 0$. Certainly, this global estimate is not enough for the proof of theorem 2 , which is an essentially stronger estimate $\left\|\rho\left(\tilde{\mathbf{1}}_{\varepsilon}\right)\right\|_{\mathcal{L}^{\infty}(\mathbb{L})}=O(1)$ on the lines $\mathbb{L}=\mathbb{L}_{N, \frac{M}{2}}$ or $\mathbb{L}=\mathbb{L}_{N, \frac{M}{2}}^{T}$ with odd $N, M$. According to (33), the following lemma is sufficient.

Lemma 3. Assume that $N, M$ are odd integers, $\varepsilon \in\left(0, \frac{1}{|N|}\right)$. Then

$$
\begin{aligned}
& \sup _{(t, x) \in \mathbb{L}_{N, \frac{M}{2}}}|H(t, x+\varepsilon)-H(t, x-\varepsilon)| \leq c \sqrt{\varepsilon} \\
& \sup _{(t, x) \in \mathbb{L}_{N, \frac{M}{2}}^{T}}|H(t, x+\varepsilon)-H(t, x-\varepsilon)| \leq c \sqrt{|N| \varepsilon} .
\end{aligned}
$$

We only outline the proof. For $H(\mathbf{x})=H(t, x)$ more precise estimates are known, see [26], [28], than the general self-similarity relation (32). We have

$$
\mathcal{H}(t, x)=\text { p.v. } \int_{R^{1}} \frac{e^{2 \pi i\left(n^{2} t+n x\right)}}{2 \pi i n} d n=e^{\frac{\pi i}{4}} \int_{0}^{\frac{x}{\sqrt{2 t}}} e^{-\pi i \xi^{2}} d \xi, \quad t>0,
$$

i.e. the integral Hilbert transform is expressed by the incomplete Fresnel's integral (if $t<0$, one can find the value of $\mathcal{H}$ using a general relation $\left.\mathcal{H}(-\mathbf{x})=\mathcal{H}^{*}(\mathbf{x})\right)$. Further, the following estimates are valid in the neighborhood of a rational point $\mathbf{y}=\left(\frac{a}{q}, \frac{m}{q}\right) \in$ $\mathbb{Q}^{2}, q=q(\mathbf{y})$,

$$
\begin{aligned}
& H(\mathbf{y}+\mathbf{z})-H(\mathbf{y}+\tilde{\mathbf{z}})=G(\mathbf{y})(\mathcal{H}(\mathbf{z})-\mathcal{H}(\tilde{\mathbf{z}}))+O\left(\sqrt{q}\left(\sqrt{\left|z_{2}\right|+\left|\tilde{z}_{2}\right|}+\left|z_{1}-\tilde{z}_{1}\right|\right)\right), \\
& \mathbf{y}+\mathbf{z}, \mathbf{y}+\tilde{\mathbf{z}} \in \tilde{\square}^{2}(\mathbf{y}), \quad \tilde{\square}^{2}(\mathbf{y}):=\left\{\mathbf{y}+\mathbf{z},\left|z_{2}\right| \leq \frac{10}{q^{2}} ; \quad\left|z_{1}\right| \leq \frac{1}{2 q}\right\} .
\end{aligned}
$$

Lemma 3 is deduced from these relations by the arguments close to the proof of lemma 2. The main difference is that instead of (29), here we utilize the following estimate of the differences of the Fresnel integral $\mathcal{H}$ :

$$
\begin{aligned}
& \left|\int_{a}^{b} e^{\pi i \xi^{2}} d \xi\right|=\left|\int_{a}^{b} \frac{d\left(e^{\pi i \xi^{2}}\right)}{2 \pi i \xi} d \xi\right| \leq \frac{1}{\pi a}, \quad 1 \leq a \leq b ; \\
& \left|H\left(z_{2}, \tilde{z}_{1}\right)-H\left(z_{2}, z_{1}\right)\right| \leq c \min \left(1, \frac{\sqrt{\left|z_{2}\right|}}{z_{1}}\right), \quad 0 \leq z_{1} \leq \tilde{z}_{1} .
\end{aligned}
$$


9. Weak quadratic variation, local properties of the solutions. Relation (34) was utilized in [26] to establish that the Hilbert transform $H(t, x)$, as a function of the variable $x$ for fixed $t$, is of bounded weak quadratic variation (weak 2-variation) on the period $[0,1)$, and this property holds uniformly in $t$ :

$$
\sup _{t \in \mathbb{R}^{1}}\|H(t, \cdot)\|_{\mathcal{W} \mathcal{V}_{2}\left(\mathbb{T}^{1}\right)}<\infty .
$$

For a fixed number $p \geq 1$, the definitions of the weak $p$-variation, and the corresponding norm $\|\cdot\|_{\mathcal{W} \mathcal{V}_{p}\left(\mathbb{T}^{1}\right)}$ of a (bounded) periodic function $h: \mathbb{T}^{1} \rightarrow \mathbb{C}$, are the following. For a given $N \in \mathbb{N}$, denote by $\mathcal{C}_{N}\left(\mathbb{T}^{1}\right)$ the set of all periodic piecewise constant functions with $N$ intervals of constancy on $\mathbb{T}^{1}$, i.e., $g \in \mathcal{C}_{N}$ if there exist $N$ intervals $I_{j}=\left[a_{j}, a_{j+1}\right), j=1, \ldots, N$, such that $a_{N+1}-a_{1}=1$, and the value of $g$ is constant on each $I_{j}$. Further, denote

$$
\sigma_{N}(h):=\inf _{g \in \mathcal{C}_{N}\left(\mathbb{T}^{1}\right)}\|h-g\|_{\mathcal{L}^{\infty}\left(\mathbb{T}^{1}\right)}
$$

the value of the best $N$-term non-linear approximation of $h$ in $\mathcal{L}^{\infty}$ by piecewise constant functions. We say that $h$ is of bounded weak $p$-variation ${ }^{6}$ on $\mathbb{T}^{1}$, if $\sigma_{N}(h)=$ $O\left(N^{-1 / p}\right), N \rightarrow \infty$. Let $\operatorname{wvar}_{p}\left(h, \mathbb{T}^{1}\right):=\sup _{N \in \mathbb{N}} N^{1 / p} \sigma_{N}(h)$, and denote by $\mathcal{W} \mathcal{V}_{p}\left(\mathbb{T}^{1}\right)$ the space of all functions $h$ of bounded weak $p$-variation on $\mathbb{T}^{1}$, with the norm

$$
\|h\|_{\mathcal{W} \mathcal{V}_{p}\left(\mathbb{T}^{1}\right)}:=\operatorname{wvar}_{p}\left(h, \mathbb{T}^{1}\right)+|f(0)| .
$$

A corollary of (35) is the variational property of the solution operator $\psi$ of (1):

$$
\sup _{t \in \mathbb{R}^{1}}\|\psi(f ; t, \cdot)\|_{\mathcal{W} \mathcal{V}_{2}\left(\mathbb{T}^{1}\right)} \leq c\|f\|_{\mathcal{B V}\left(\mathbb{T}^{1}\right)} .
$$

Let us note, that it is not possible to improve the above variational result essentially: one cannot take the strong quadratic variation (in the sense of $\mathrm{N}$. Wiener) instead of its weak version, as it was defined above, see [26].

Below are listed some other properties of the function $H(x, t)$ and the solution $\psi(f ; t, x)$ (we assume that $f \in \mathcal{B V}\left(\mathbb{T}^{1}\right)$ ), see [28], or [24], p. 222.

A) If $t$ is a fixed irrational number, then the solution $\psi(f ; t, x)$ is a continuous function of the variable $x$; the function $H(\mathbf{x})=H(t, x)$ is continuous, but not differentiable for almost all $x$. $H$ is discontinuous at all rational points $\mathbf{y} \in \mathbb{Q}^{2}$ where $G(\mathbf{y}) \neq 0$.

B) If the sequence of the partial quotients $\left\{k_{j}\right\}$ of the continued fraction (24), is bounded (in particular, if $t$ is a quadratic irrationality, like $t=\sqrt{2}$ ) then the solution $\psi$ satisfies the Lipschitz-Hölder condition of order $1 / 2$, i.e.

$$
\omega(\psi(f ; t, \cdot), \delta)=O\left(\delta^{1 / 2}\right), \delta \rightarrow 0 ; \quad \omega(h(\cdot), \delta):=\sup _{|x-y| \leq \delta}|h(x)-h(y)|, \delta>0 .
$$

For almost every fixed $t$, the following estimate of the uniform modulus of continuity of the solution is true:

$$
\omega(\psi(f ; t, \cdot), \delta)=O\left(\delta^{1 / 2}|\ln \delta|^{1 / 4+\varepsilon}\right), \quad \forall \varepsilon>0, \delta \rightarrow 0 .
$$

\footnotetext{
${ }^{6}$ In [26], another definition of the weak $p$-variation was used, namely, via counting "large" oscillations of $h$ over all arbitrary partitions of the period. However, the alternative definition is equivalent to the given above.
} 
C) The solution $\psi(f ; t, x)$ is continuous on every line, non-parallel to the $x$-axis, and in particular, $\psi$ is a continuous function of the variable $t$ for each fixed $x$.

REMARK 5. The latter property means that for the initial data of the class $\mathcal{B V}\left(\mathbb{T}^{1}\right)$, the time-evolution described by (1) is continuous. In this sense, $\psi$ behaves better than the solution $u$ of the linearized KdV-equation (9). The solution operator $u$ of (9) transfers one singularity of the initial data $f(x)=\frac{1}{2}-\{x\}$ on $\mathbb{T}^{1}$ to a countable everywhere dense set of singularities (jumps) of the solution

$$
u(f, t, 0)=A(t)=\sum_{n \in \mathbb{N}^{1}} \frac{\sin 2 \pi n^{3} t}{\pi n}
$$

as a function of $t$.

10. An application to the incomplete Gauss sums. Let $q \in \mathbb{N}, a \in \mathbb{Z},(a, q)=1$, and let $I$ be an interval on $\mathbb{R}^{1}$ whose length satisfies $|I|<q-1$. Then the expression

$$
S\left(\frac{a}{q}, I\right):=\sum_{n \in I} e^{\frac{2 \pi i a n^{2}}{q}}
$$

is called the incomplete Gauss sum, corresponding to the parameter $t=\frac{a}{q}$ and the interval $I$.

The incomplete Gauss sums satisfy the estimate

$$
\sup _{|I| \leq q-1}\left|S\left(\frac{a}{q}, I\right)\right| \leq c \sqrt{q} .
$$

This estimate is essentially due to G.H. Hardy and J. E. Littlewood [15], although it has not been explicitly mentioned by the authors. E.C. Titchmarsh included this estimate into the comments concerning [15], see [16], v. 1, p. 113 - 114; see also [13].

As a matter of fact, the estimate (36) is equivalent to the statement $H \in \mathcal{L}^{\infty}\left(\mathbb{T}^{2}\right)$. Moreover, $H(t, x)$ is a generating function, it "encodes" all possible incomplete Gauss sums, see [26].

In this relation, it may be interesting to recall a comment made by $\mathrm{S}$. Chowla, see [9], v. 1, pp. $426-428$. This paper discusses the unboundedness problem for the sums $\sum_{1}^{r} e^{i \alpha n^{2}} \frac{\sin n \beta}{n}$. A footnote on the first page mentions that the formulation of the latter problem is due to H. Davenport and H. Heilbronn. Secondly, it is remarked that the problem has been solved in the negative by Dr. Spaček of Prague. The latter amounts to the statement that indeed $H \in \mathcal{L}^{\infty}\left(\mathbb{T}^{2}\right)$. However, the author of the present paper did not succeed in locating the publications by Davenport, Heilbronn and Spaček on this issue.

Utilizing the weak quadratic variation result (35), it is possible to complement the estimate (36) as follows, cf. [26]. If $\left\{I_{j}\right\}$ is an arbitrary collection of pairwise non-intersecting intervals on $[1, q]$, then

$$
\operatorname{card}\left\{\left|S\left(\frac{a}{q}, I_{j}\right)\right| \geq \varepsilon \sqrt{q}\right\} \leq c \varepsilon^{-2}, \varepsilon>0,
$$

where card $\{\cdot\}$ denotes the number of elements of a (finite) set $\{\cdot\}$, and $c$ is an absolute 
positive constant. In particular

$$
\sum_{j}\left|S\left(\frac{a}{q}, I_{j}\right)\right|^{\alpha} \leq c_{\alpha} q^{\alpha / 2}, \quad \alpha>2,
$$

where the factor $c_{\alpha}$ depends only on $\alpha$, and is finite for $\alpha>2$.

11. Some functional identities, and inequalities. The "imaginary heat transfer kernel"

$$
\Gamma(t, x)=\text { p.v. } \int_{\mathbb{R}^{1}} e^{\pi i\left(y^{2} t+2 y x\right)} d y=\sqrt{\frac{i}{t}} e^{-\frac{\pi i x^{2}}{t}}, \quad t \neq 0, \quad \sqrt{i}:=e^{\frac{\pi i}{4}}
$$

is the Green function for the Cauchy problem

$$
\frac{\partial \varphi}{\partial t}=\frac{1}{4 \pi i} \frac{\partial^{2} \varphi}{\partial x^{2}},\left.\quad \varphi(f ; t, x)\right|_{t=0}=f(x)=\int_{\mathbb{R}^{1}} \hat{f}(y) e^{2 \pi i y x} d y
$$

with a general, not necessarily periodic, initial data $f ; \hat{f}$ denotes the Fourier transform of $f$ on $\mathbb{R}^{1}$, and both $f, \hat{f}$ can be understood in generalized sense, as tempered distributions. Comparing two representations of the solution operator, via the convolution of $f$ with the Green function $\Gamma$, and the direct, via the Fourier separation of variables, one obtains, cf. [27], the following general functional identity

$$
\varphi(f ; t, x)=\sqrt{\frac{i}{t}} e^{-\frac{\pi i x^{2}}{t}} \varphi\left(\hat{f} ;-\frac{1}{t},-\frac{x}{t}\right), \quad \sqrt{i}:=e^{\frac{\pi i}{4}} .
$$

This identity relates the solution $\varphi(\hat{f})$ of the problem $(37)$ with the solution of the same problem, but posed for the Fourier transform $\hat{f}$ as the initial data. It is of the well-known type, of course, namely, a variant of the classical functional equation for the Jacobi elliptic $\vartheta$-function. If we take the periodic delta-function $f=\sum_{n \in \mathbb{Z}} \delta_{n}$ as the initial data, then $\hat{f} \equiv f$ (the Poisson summation formula), and we obtain from (38) the following particular functional identity

$$
\Theta(t, x)=\sqrt{\frac{i}{t}} e^{-\frac{\pi i x^{2}}{t}} \Theta\left(-\frac{1}{t},-\frac{x}{t}\right), \quad \Theta(t, x):=\sum_{n \in \mathbb{Z}} e^{\pi i\left(n^{2} t+2 n x\right)}, \quad t \neq 0 .
$$

This identity is meaningful only as a relation between two distributions, or generalized functions. For fixed rational values of $t=\frac{a}{q}, \Theta$ as a function of $x$ is " atomic", i.e. the sum of Dirac's $\delta$-functions with the complex coefficients. In fact, (39) is equivalent to the well-known identity of Genocchi and Schaar (see [14], pp. 226 - 227) for the bisected Gauss sums

$$
\sqrt{\frac{1}{q}} \sum_{n=1}^{q} e^{\frac{\pi i a n^{2}}{q}}=\sqrt{\frac{i}{a}} \sum_{m=1}^{a} e^{-\frac{\pi i q n^{2}}{a}}, \quad a, q \in \mathbb{N}^{1},(a, q)=1, a-q \equiv 1(\bmod 2) .
$$

An integration of both sides of (39) in the variable $x$ results in a pointwise functional identity for the values of the function

$$
h(t, x):=H\left(\frac{t}{2}, x\right)=\text { p.v. } \sum_{n \in \mathbb{Z} \backslash\{0\}} \frac{e^{\pi i\left(n^{2} t+2 n x\right)}}{2 \pi i n} .
$$


As a conclusion, it was shown in [27] that the following functional inequality is valid for the function $h$ :

$$
|h(t, x)| \leq \sqrt{t}\left|h\left(\frac{1}{t}, \frac{x}{t}\right)\right|+c, \quad(t, x) \in \square_{0}:=(0,1) \times\left[-\frac{1}{2}, \frac{1}{2}\right],
$$

where $c$ is an absolute constant. One has $h(t+1, x) \equiv h(t, x+1 / 2) ; h(t, x+1) \equiv h(t, x)$. Therefore, representing $\frac{1}{t}$ as $\frac{1}{t}=\left[\frac{1}{t}\right]+\tau, \tau:=\left\{\frac{1}{t}\right\}$, one can iterate (40), keeping the new variables $(\tau, \xi)$ under control, i.e. in the rectangle $\square_{0}$. This "dynamical process" of iterations of (40) results in an alternative proof that $H \in \mathcal{L}^{\infty}\left(\mathbb{R}^{2}\right)$, and let us note that in this approach, almost all number-theoretical complications are eliminated. Let us also note that the idea of utilizing iterations of functional identities, or the approximate identities in the study of $\Theta$ appears in many places in the papers of Hardy and Littlewood [14], [15]. In particular, they established the following approximate functional equation for the partial sums of $\Theta$ :

$$
\Theta_{n}(t, x)=\sqrt{\frac{i}{t}} e^{-\frac{\pi i x^{2}}{t}} \Theta_{n t}^{*}\left(\frac{1}{t}, \frac{x}{t}\right)+O\left(\frac{1}{\sqrt{t}}\right), \quad \Theta_{n}(t, x):=\sum_{1 \leq \nu \leq n} e^{2 \pi i\left(\nu^{2} t+2 n x\right)} .
$$

Finally, let us note that the density $\rho(f)$ also satisfies the functional equation

$$
\rho(f, t, x)=\frac{1}{2|t|} \rho\left(\hat{f},-\frac{1}{2 t},-\frac{x}{2 t}\right)
$$

which is the corollary of $(38)$.

However, it seems more interesting to look on the density as the trace of the solution $\left.R(t, x, y)\right|_{y=0}$ of the Cauchy initial value problem of the following bivariate Schrödinger type equation:

$$
\frac{\partial R}{\partial t}=\frac{1}{2 \pi i} \frac{\partial^{2} R}{\partial x \partial y},\left.\quad R(F, t, x, y)\right|_{t=0}=F(x, y):=f(x+y) f^{*}(x-y) .
$$

The solution $R$ of this problem for a general $F$ via the Fourier method of separation of variables is given by the double oscillatory integral (or sum, if the initial data function $F$ is bi-periodic) with the hyperbolic phase

$R(F, t, x, y)=\int_{\mathbb{R}^{2}} \hat{F}(\xi, \eta) e^{2 \pi i(\xi \eta t+\xi x+\eta y)} d \xi d \eta, \quad \hat{F}(\xi, \eta)=\int_{\mathbb{R}^{2}} F(x, y) e^{-2 \pi i(\xi x+\eta y)} d x d y$, and the Green function is

$$
G(t, x, y)=\frac{e^{-\frac{2 \pi i x y}{t}}}{|t|} .
$$

The analog of the functional identity (38) for the solution operator $R$ is the following:

$$
R(F,(t, x, y))=\frac{e^{-\frac{2 \pi i x y}{t}}}{|t|} R\left(\hat{F},-\frac{1}{t}(1, y, x)\right) .
$$

If the bivariate initial data function $F$ is periodic in both variables $x, y$, of period $=1$, the (generalized) solution of the problem (41) is represented by the double trigonometric series with the hyperbolic phase

$$
R(F, t, x, y)=\sum_{(n, m) \in \mathbb{Z}^{2}} \hat{F}_{n, m} e^{2 \pi i(n m t+n x+m y)} .
$$


Not too much is known concerning the properties of the sums of such series, nor their convergence in non-trivial cases. That the sums of such series are self-similar, has been demonstrated above, by the elaboration of the case of the initial data $F$ of the type

$$
F(x, y)=f(x+y) f^{*}(x-y), \quad \hat{F}_{n, m}=\hat{f}_{\frac{n+m}{2}} \hat{f}_{\frac{n-m}{2}}^{*},
$$

and the trace of the solution $\left.R(f, t, x, y)\right|_{y=0}$ is the density function $\rho(f, t, x)$. St. Jaffard [20] studied the convergence, and the multi-fractal properties of the sums, of an interesting class of Davenport expansions. The latter are the series of the type $\sum_{n} a_{n}(\{n t\}-1 / 2)$, where as above, we keep the notation $\{\cdot\}$ for the fractional part function. Such series first appeared in the works of H. Davenport [11], [12]. One can also look on the Davenport expansions as the traces $\left.R(t, x, y)\right|_{x=y=0}$ of the solution $R$ of the Cauchy problem (41) for the initial data function $F$ of the separable type

$$
F(x, y)=A(x)\left(\{y\}-\frac{1}{2}\right), \quad A(x):=\sum_{n} a_{n} e^{2 \pi i n x}, \quad \hat{F}_{n, m}=\frac{a_{n}}{2 \pi i m}, \quad m \neq 0 .
$$

In a recent paper [29], the author studied the convergence of the double trigonometric series

$$
C_{1}(t):=\sum_{(n, m) \in \mathbb{N}^{2}} \frac{\sin 2 \pi n m t}{\pi n m}, \quad C_{2}(t):=\sum_{(n, m) \in \mathbb{N}^{2}} \frac{\cos 2 \pi n m t}{\pi n m} .
$$

These series are also traces of the $R$-function. The convergence sets, in a rather wide understanding of the summation process over expanding families of the domains on $\mathbb{N}^{2}$, coincides, respectively, with the convergence sets of the univariate series

$$
\Xi_{1}(t):=\sum_{j=0}^{\infty} \frac{(-1)^{j} \ln q_{j+1}}{q_{j}}, \quad \Xi_{2}(t):=\sum_{j=0}^{\infty} \frac{\ln ^{2} q_{j+1}}{q_{j}},
$$

where $q_{j}=q_{j}(t)$ denotes the denominator of the $j$ th convergent fraction $\frac{a_{j}}{q_{j}}$ of a given real $t$, see (24). Such recursive series, defined by the continued fraction of $t$, were introduced by J. R. Wilton [39]. Wilton established in terms of $\Xi_{1}, \Xi_{2}$ the full characterization of the convergence sets of the series

$$
\sum_{n=1}^{\infty} \frac{d(n) \sin 2 \pi n t}{\pi n}, \quad \sum_{n=1}^{\infty} \frac{d(n) \cos 2 \pi n t}{\pi n},
$$

where $d(n)=\sum_{m \mid n} 1$ denotes the divisor function. The latter univariate series correspond to the summation of the the double series $C_{1}, C_{2}$ over expanding families of hyperbolic crosses on $\mathbb{N}^{2}$. A further development of Wilton's result is contained in a recent paper of R. de la Bretèche and G. Tenenbaum [7]. The author of the present paper is indebted to G. Tenenbaum for bringing up the reference [39].

Acknowledgements. The author work was supported by the Grant DMS 0410012 of the National Science Foundation of the USA.

The author extends his deep gratitude to M. Benedicks, A. Bobylev, L. Carleson, D. Dix, V. Gudkov, S. Jaffard, M. Lacey, E. Kopecka, M. Matsutani and Z. Ciesielski for their interest, useful comments and discussions. 
The author also wishes to thank the referee of this paper for a number of valuable remarks and suggestions.

\section{References}

[1] G. I. Arkhipov, On the Hilbert-Kamke problem, Izv. Akad. Nauk SSSR Ser. Mat. 48 (1984), 3-52; English transl. in Math. USSR Izv. 24 (1985).

[2] G. I. Arkhipov and K. I. Oskolkov, On a special trigonometric series and its applications, Matem. Sbornik 134 (176) (1987), no. 2; English transl. in Math. USSR Sbornik 62 (1989), $145-155$.

[3] M. V. Berry, Quantum fractals in boxes, J. Physics A: Math. Gen 29 (1996), 6617-6629.

[4] M. V. Berry and S. Klein, Integer, fractional and fractal Talbot effects, J. Mod. Optics 43 (1996), 2139-2164.

[5] M. Berry, I. Marzoli and W. Schleich, Quantum carpets, carpets of light, Physics World, June 2001, 1-6.

[6] D. Bohm, Quantum Theory, Dover Publications, New York.

[7] R. de la Bretèche et G. Tenenbaum, Séries trigonométriques à coefficients arithmétiques, J. d'Analyse Math. 92 (2004), 1-79.

[8] L. Carleson, Some analytic problems related to statistical mechanics, in: Euclidean Harmonic Analysis (College Park, Md., 1979), Lecture Notes in Math. 779, Springer-Verlag, 1980, 5-45.

[9] The collected papers of Sarvadaman Chowla, J. G. Huard and Kenneth S. Williams (eds.), Les Publ. CRM, Vols. 1-3.

[10] B. E. J. Dahlberg and C. E. Kenig, A note on the almost everywhere behavior of solutions to the Schrödinger equation, in: Harmonic Analysis (Minneapolis, Minn., 1981), Lecture Notes in Math. 908, Springer-Verlag, 1982, 205-209.

[11] H. Davenport, On some infinite series involving arithmetical functions, Quarterly Journal of Mathematics 8 (1937), 8-13.

[12] H. Davenport, On some infinite series involving arithmetical functions II, Quarterly Journal of Mathematics 8 (1937), 313-320.

[13] H. Fiedler, W. Jurkat and O. Koerner. Asymptotic expansion of finite theta series, Acta Arithmetica 32 (1977), 129-146.

[14] G. H. Hardy and J. E. Littlewood, Some problems of Diophantine approximation, in: Proceedings of the 5th International Congress of Mathematicians, Cambridge, 1912, 223229 .

[15] G. H. Hardy and J. E. Littlewood, Some problems of Diophantine approximation. II. The trigonometric series associated with the elliptic $\vartheta$-functions, Acta Mathematica 37 (1914), 193-238.

[16] Collected papers of G. H. Hardy, Including joint papers with J. E. Littlewood and others, Oxford University Press, 1966.

[17] P. R. Holland, The Quantum Theory of Motion. An Account of the de Broglie-Bohm Causal Interpretation of Quantum Mechanics, Cambridge University Press.

[18] H. L. Keng, Introduction to Number Theory, Springer-Verlag, Berlin, 1982.

[19] A. D. Ionescu and S. Wainger, $L^{p}$ boundedness of discrete singular Radon transforms,

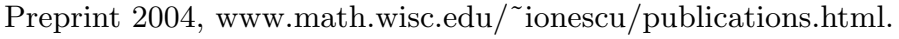


[20] S. Jaffard, On Davenport expansions, in: Proceedings of Symposia in Pure Mathematics 72, 273-303.

[21] C. E. Kenig and A. Ruiz, A strong type $(2,2)$ estimate for a maximal operator associated to the Schrödinger equation, Trans. Amer. Math. Soc. 280 (1983), 239-246.

[22] S. Matsutani and Y. Ônishi. Wave-particle complementarity and reciprocity of Gauss sums on Talbot effects, Foundations of Physics Letters 16 (2003), 325-341.

[23] K. I. Oskolkov, On spectra of uniform convergence, Dokl. Akad. Nauk SSSR 288 (1986), no 1 (in Russian). English translation in: Soviet Math. Dokl. 33 (1986), no.3, 616-620.

[24] K. I. Oskolkov, Series and integrals of I. M. Vinogradov and their applications, Trudy Matem. Inst. Steklova 190 (1989) (in Russian). English translation in: Proceedings of the Steklov Institute of Mathematics, 1992, Issue 1, 193-229.

[25] K. I. Oskolkov, Vinogradov series in the Cauchy problem for equations of Schrödinger type, Trudy Matem. Inst. Steklova 200 (1991) (in Russian). English translation in: Proceedings of the Steklov Institute of Mathematics, 1993, Issue 2, 291-315.

[26] K. I. Oskolkov, On functional properties of incomplete Gaussian sums, Canadian Journal of Mathematics 43 (1991), 182-212.

[27] K. I. Oskolkov, Schrödinger equation and oscillatory Hilbert transforms of second degree, J. Fourier Analysis and Applications 3 (1998), 341-356.

[28] K. I. Oskolkov, A class of I. M. Vinogradov's series and its applications in harmonic analysis, in: Progress in Approximation Theory: an International Perspective, A. A. Gonchar and E. B. Saff (eds.), Springer-Verlag, 353-402.

[29] K. I. Oskolkov, The series $\sum \sum \frac{e^{2 \pi i m n x}}{m n}$, and a problem of Chowla, Trudy Matematicheskogo Instituta imeni V. A. Steklova 248, 204-222 (in Russian); English translation in: Proc. Steklov Inst. Math. 248, 197-215.

[30] P. P. Petrushev, Approximation by ridge functions and neural networks, SIAM Journal on Mathematical Analysis 30 (1998), 155-189.

[31] W. P. Schleich, Quantum Optics in Phase Space, Wiley-VCH, Berlin, 2001.

[32] P. Sjölin, Regularity of solutions to the Schrödinger equation, Duke Math. J. 55 (1987), 699-715.

[33] E. M. Stein and S. Wainger. The estimation of an integral arising in multiplier transformations, Studia Math. 35 (1970), 101-104.

[34] E. M. Stein and S. Wainger, Discrete analogs of singular Radon transforms, Bull. Amer. Math. Soc. 23 (1990), 537-544.

[35] W. H. F. Talbot, Facts relating to optical sciences. IV, Philosophical Magazine 9 (1838), 401-407.

[36] P. L. Ul'yanov, Some problems in the theory of orthogonal and biorthogonal series, Izv. Akad. Nauk Azerb. SSR, Ser. Fiz.-Tekhn. Mat. Nauk (1965), no. 6, 11-13.

[37] I. M. Vinogradov, The Method of Trigonometric Sums in Number Theory, 2nd ed., Nauka, Moscow, 1980; English transl. in his Selected Works, Springer-Verlag, 1985.

[38] H. Weyl, Über die Gleichverteilung von Zahlen mod. Eins, Mathematische Annalen 77 (1916), 313-352.

[39] J. R. Wilton, An approximate functional equation with applications to a problem of Diophantine approximation, J. Reine Angew. Math. 19 (1933), 219-237.

[40] A. Zygmund, Trigonometric Series, Cambridge University Press, second edition, 1959. 